\title{
A General Theory of Polymer Ejection Tested in a Quasi Two-Dimensional Space
}

Pai-Yi Hsiao ( $\square$ pyhsiao@ess.nthu.edu.tw )

National Tsing Hua University

Wei-Yei Chen

National Tsing Hua University

\section{Research Article}

Keywords: polymer science, nanoscience, polymer ejection

Posted Date: May 13th, 2021

DOl: https://doi.org/10.21203/rs.3.rs-514614/v1

License: (c) (i) This work is licensed under a Creative Commons Attribution 4.0 International License. Read Full License

Version of Record: A version of this preprint was published at Scientific Reports on July 19th, 2021. See the published version at https://doi.org/10.1038/s41598-021-94054-2. 


\title{
A General Theory of Polymer Ejection Tested in a Quasi Two-Dimensional Space
}

\author{
Pai-Yi Hsiao ${ }^{1,2, *}$ and Wei-Yei Chen ${ }^{1}$ \\ ${ }^{1}$ Department of Engineering and System Science, \\ National Tsing Hua University, Hsinchu, Taiwan, R.O.C. \\ ${ }^{2}$ Institute of Nuclear Engineering and Science, \\ National Tsing Hua University, Hsinchu, Taiwan, R.O.C. \\ *Corresponding author, email: pyhsiao@ess.nthu.edu.tw; pyhsiao@mx.nthu.edu.tw
}

(Dated: May 12, 2021) 


\begin{abstract}
A general ejection theory of polymer is developed in a two- and three-dimensional space. A polymer is confined initially in a cavity and ejects spontaneously to the outer space through a nanopore channel without the help of any external stimulus. A reflective wall boundary is set at the pore entrance to prevent the falling of the head monomer of chain into the cavity. Three stages are distinguished in a process: (1) the entering stage, in which the head monomer enters the pore to search for a way to traverse the pore channel, (2) the main ejection stage, in which the chain body is transported from the cavity to the outer space, (3) the leaving stage, in which the tail monomer passes through and leaves the pore channel. Depending on the number of the monomers remaining in the cavity, the main ejection stage can be divided into the confined and the non-confined stages. The non-confined stage can be further split into the thermal escape and the entropic pulling stages. The Onsager's variational principle is used to derive the kinetics equation of ejection. The escape time is calculated from the corresponding Kramers' escape problem. Extensive molecular dynamics simulations are then performed in a quasi two-dimensional space to verify the theory. The variation of the ejection speed is carefully examined in a process. The decreasing behavior of the number of monomers in the cavity is studied in details. The scaling properties of the spending time at each processing stage are investigated systematically by varying the chain length, the cavity diameter, and the initial volume fraction of chain. The results of simulation support firmly the predictions of the theory, cross-checked in the studies of various topics. Together with the previous investigations in the three-dimensional space, the generalized theory is very robust able to explain the two seemly different phenomena, polymer ejection and polymer translocation, under the same theoretical framework in the two space dimensions.
\end{abstract}

\title{
I. INTRODUCTION
}

One of the main objectives in bionanotechnology is to develop efficient techniques for control or manipulation of biomacromolecules for diverse applications [1 3]. Recent advances have demonstrated the capabilities to imprison or encapsulate single DNA or RNA chains in a small trap or cavity for preserving or transportation [4 7]. The molecular chains are released at the moment of necessary to a destination space for sensing or therapeutic purposes [8, 9] In Nature, bacteriophage is a virus mastering the skill [10, 11]. It infects bacteria by 
ejecting the genetic materials encapsulated in the viral capsid through a small channel into the host cell for replication. The ejecting process occurs spontaneously where no external driving force intervenes in pressing or pulling the chain out of the cavity. It is a result of the favor of thermodynamics because the chemical potential inside the capsid is higher than the outside [12, 13].

The ejecting problem takes place at nanometer scales where the thermal fluctuations have great influences. The stochastic nature of the surroundings blurs the deterministic trajectory of ejection issued from the confinement. It renders the classical method, by solving the problem under the framework of continuum mechanics, insufficient in describing the properties of such a process [12 20]. External factors such as temperature [21], acidity of environment [22], ions and salinity [23], osmotic pressure [20, 24], etc., can disturb the progress of ejection [25]. Other effects like protein binding in the cytoplasm of host cell [18, 21] and condensation of the ejected chain [26] can help the ejection. The factors and effects complicate the process and make the system difficult to be predicted.

A fundamental approach to study the ejection problem at a primitive level starts with the calculation of the change of the free energy [27 32]. The ejection of chain results in a decrease of the free energy, which drives the chain out of the cavity. Polymer physics have provided a sophisticated way to estimate chain free energy under diverse situations via scaling analysis [33 35]. By adopting the free energy of polymer in a semidilute solution, Muthukumar [36] predicted that the ejection should be $\tau_{\text {ej }} \sim N^{1+(1 / 3 \nu)} \phi_{0}^{-1 / 3 \nu}$ with $\nu$ being the Flory exponent, where the initial volume fraction of chain in the cavity is $\phi_{0}$ and the chain length is $N$. Cacciuto and Luijten [37] reported later in the Monte Carlo simulations that $\tau_{\text {ej }}$ should be $N^{1+\nu} \phi_{0}^{-1 /(3 \nu-1)}$. Sakaue and Yoshinaga [38] made a careful study and pointed out an important fact that the volume fraction of the number of the monomers in the cavity decreases and thus, the driving chemical potential difference is not constant but decreases with time. They balanced the rate of the free energy change with the rate of the energy dissipation taking place in the solution near the pore within a correlation length $\xi$; an ejection time $\tau_{\mathrm{ej}} \sim N^{(2+\nu) /(3 \nu)} \phi_{0}^{-(2+\nu) /(3 \nu)}$ was predicted. They also envisaged that the ejection process was ended by a diffusion-like escape with the spending time $\tau_{\mathrm{D}} \sim D^{2} / \mathfrak{D}_{N_{0}}$ where $D$ is the cavity diameter and $\mathfrak{D}_{N_{0}}$ the diffusion coefficient to diffuse the last segments of chain out of the cavity.

Recently, we have modified and extended the theory [39, 40]. The new theory balanced 
the rate of the free energy change with the rate of the energy dissipation at the pore with a fraction coefficient varying during the process. Instead of treating the final escape in a diffusive way, we dealt with it as a formal translocation, with the free energy being the sum of the free energies from the two chain segments instantaneously expanding on the two sides of the pore. The ejection time $\tau_{\mathrm{ej}} \sim N^{(2+\nu) /(3 \nu)} \phi_{0}^{-2 /(3 \nu)}$ was obtained in our theory. The results have been demonstrated correct by extensive molecular dynamics simulations. A double verification has been done under the $D$-fixed condition, which revealed a $N^{1 / 3}$-dependence for $\tau_{\text {ej }}$, following our prediction. Ref. [38], in contrast, expected a null dependence on $N$. Because the translocation theory is involved in the derivation, our theory successfully connects the two seemly different problems together, polymer ejection and polymer translocation [35, 41. 43]. The theory is applicable in the entire $\left(N, D, \phi_{0}\right)$ parameter space. Moreover, we pinpointed the existence of a nucleation-like stage occurred prior to the main ejection, which takes care of the traversing of the heading monomers across the pore channel. A theory has been developed by regarding it as a Kramers' escape problem and explained it in analogy to the nucleation phenomena [40].

Despite of the above achievements, there are still several issues to be clarified or resolved. For example, how does the free energy landscape look like in term of the ejection coordinate under different ejection conditions? A good knowledge of the free energy landscape enables a well understanding of the physical pictures behind an ejection phenomenon. In the theory, we have divided the main ejection into the confined and the non-confined stages, and assumed that it is the entropic pulling which drives the chain out of the cavity in the latter stage. However, it is not always the case. A situation can happen if the chain length is not long compared to the cavity diameter — the segment length in the cavity can be still longer than the one from the outside at the moment when the process enters to the non-confined stage, and therefore, the driving force is not entropic at that moment. How shall we fix the problem? Is this particular situation detectable in simulations and what is its significance? In a real world, the encapsulating cavity may not be spherical. Researchers may enclose a biopolymer in a quasi-two-dimensional cage and release it later though a pore or a canal for applications. It addresses an important question, how to generalize the current theory for a two-dimensional space. The generalization can be served as a test of the robustness of the theory.

In this paper, a general theory for polymer ejection in a $d$-dimensional space is given in 
Section II. An extensive molecular dynamics simulation is then performed in a quasi twodimensional space to verify the generalization of the theory where the modeling and settings are described in Section III. The results are reported in Section IV. The studied topics include the variation of the ejection speed during a process (Section IV A), the decrease of the number of the monomers in a confining cavity (Section IV B), and the scaling behaviors of the decomposed time as a function of $N, D$, and $\phi_{0}$ in each stage (Section IV C). The discussions and conclusions are given in Section V.

\section{GENERAL EJECTION THEORY IN A $d$-DIMENSIONAL SPACE}

Consider the problem of a polymer ejecting in a $d$-dimensional space. The polymer is initially confined in a circular cavity and ejects through a pore channel into a semi-space. The cavity is a disk if $d=2$ and a sphere if $d=3$. The chain comprises $N$ monomers, each has a diameter $\sigma$. The monomers are linearly connected by chemical bonds of length $\sigma$ too. The circular cavity has a diameter $D$ and the pore length is $\ell_{\mathrm{p}}$. The pore diameter $d_{\mathrm{p}}$ is assumed small, which allows only one monomer to pass by the cross-section at a time. Under this setting, the initial volume fraction of monomers in the cavity is $\phi_{0}=N(\sigma / D)^{d}$. The head monomer is positioned at the entrance of the pore for starting. We assume that some mechanism exists to prevent falling of the head monomer into the interior of the cavity. Therefore, the ejection theory presented here does not include the searching of the pore entrance for the head monomer from the interior.

Extending the results of the previous study [39, 40], we generalize the scaling theory. An ejection process of polymer can be divided into three stages, namely the entering stage, the main ejection stage, and the leaving stage. The process can be described by two dependent state variables: $m$, denoting the number of monomers remaining in the cavity, and $s$, denoting the one having arrived in the semi-space. Because the pore channel allows accommodation of $m_{\mathrm{p}}$ monomers, where $m_{\mathrm{p}}=\ell_{\mathrm{p}} / \sigma$, we have $N=m+m_{\mathrm{p}}+s$. The negative $s$ state $\left(-m_{\mathrm{p}} \leq s \leq 0\right)$ occurs at the beginning, the "entering stage", when the heading monomers strive to traverse the pore channel against the chemical potential difference $\Delta \mu_{\mathrm{cp}}$ created by the osmotic pressure between the cavity and the pore. An activation energy $E_{\mathrm{a}}$ is established, which is equal to $\Delta \mu_{\mathrm{cp}}$ multiplying $m_{\mathrm{p}}$. The rate of transition, also called the

rate of escape, is described by an Arrhenius-type equation $\eta^{-1} \exp \left(-E_{\mathrm{a}} / k_{\mathrm{B}} T\right)$ where $\eta$ is the 
friction coefficient [44, 45]. A detail calculation given in Appendix A in the Supplementary Information (SI) predicts $\tau_{\text {ent }} \sim \eta \exp \left(\frac{m_{\mathrm{p}} \Delta \mu_{\mathrm{cp}}}{k_{\mathrm{B}} T}\right)$ for $m_{\mathrm{p}} \Delta \mu_{\mathrm{cp}} \gg k_{\mathrm{B}} T$. If $\Delta \mu_{\mathrm{cp}} \ll 0$, we have $\tau_{\text {ent }} \sim\left(\frac{\eta \sigma^{2}}{\mid \Delta \mu_{\mathrm{cp} \mid}}\right) m_{\mathrm{p}}$. In between $\left(\left|\Delta \mu_{\mathrm{cp}}\right|\right.$ is close 0$), \tau_{\text {ent }}$ has an expression of the diffusion time $\frac{\eta \ell_{\mathrm{p}}^{2}}{2 k_{\mathrm{B}} T}$.

The main ejection stage is started as the first monomer enters the semi-space, and ended as the last monomer leaves the cavity. In the language of the state variables, it happens when $0<s<N-m_{\mathrm{p}}$ and $N-m_{\mathrm{p}}>m>0$. The stage can be further subdivided into two stages according to the $m$ value. At the confined stage, the chain feels the confining effect of the cavity and is "compressed" out of it through the pore. As $m$ decreases and becomes eventually smaller than a critical number $N_{*}$, the remaining chain size is smaller than the cavity size. At that moment, there is no confining effect on the chain and the system is in the non-confined stage. The critical number $N_{*}$ has been shown to scale as $(D / \sigma)^{1 / \nu}$ with $\nu=3 / 5$ in the three-dimensional space [40]. We will verify it later if the scaling is truly held by switching the Flory exponent to its two-dimensional value $\nu=3 /(d+2)=3 / 4$.

The kinetics equation for the state variable $m$ can be deduced from Onsager's variational principle [40, 46, 47] and reads as

$$
\frac{\mathrm{d} m}{\mathrm{~d} t}=-\frac{1}{\eta \sigma^{2}} \frac{\mathrm{d} F}{\mathrm{~d} m}
$$

where $F$ is the free energy of the system. By using the blob theory, $F$ can be shown to be $\sim k_{\mathrm{B}} T\left(m / N_{*}\right)^{z_{1}+1}$ at the confined stage [34, 48]. Here $z_{1}=1 /(d \nu-1)$ is a key exponent appeared regularly in the des Cloizeaux theory for polymer solutions [49] and we have expressed it in the general form for a $d$-dimensional space. The kinetics equation for $m$ is thus

$$
\frac{\mathrm{d} m}{\mathrm{~d} t} \sim-\frac{z_{1}+1}{\Delta \mathfrak{t}}\left(\frac{m^{z_{1}}}{N_{*}^{z_{1}+1}}\right)
$$

where $\Delta \mathfrak{t}=\eta \sigma^{2} / k_{\mathrm{B}} T$ is the characteristic time. It has been argued that $\eta$ exhibits a $N^{x_{1}}$ dependence with $x_{1}=1 / 3$ in the three-dimensional space [39, 40]. A general formula for $x_{1}$ should be $1 / d$, which leads to $\Delta \mathfrak{t} \sim N^{1 / d} \Delta \mathfrak{t}_{0}$. Here $\Delta \mathfrak{t}_{0}=\eta_{0} \sigma^{2} / k_{\mathrm{B}} T$ and $\eta_{0}$ is the friction coefficient for a monomer. Intensive simulations will be performed later to verify it for the two-dimensional case.

At the non-confined stage, the free energy can be approximately described by

$$
F \sim k_{\mathrm{B}} T\left[\left(1-\gamma_{1}\right) \ln (m+1)+\left(1-\gamma_{1}\right) \ln (s+1)-\left(N-m_{\mathrm{p}}\right) \ln q\right]
$$


where $\gamma_{1}$ is the entropic exponent and $q$ is the effective coordination number. It is derived from $F \sim-k_{\mathrm{B}} T \ln \left(Z_{m} Z_{s}\right)$ with $Z_{m} \sim(m+1)^{\gamma_{1}-1} q^{m}$ and $Z_{s} \sim(s+1)^{\gamma_{1}-1} q^{s}$ being the partition functions for a polymer of $m$ and $s$ monomers, respectively, tethered on a surface [35, 50, 51]. The value of $\gamma_{1}$ is about 0.687 for $d=3$ [52] and 0.955 for $d=2$ [53]. To avoid the divergence of $F$ at $m=0$ and $s=0$, we have remedied the expression by adding one to the base of the exponent $\gamma_{1}-1$ in the partition function to assure $Z_{0}=1$. The kinetics equation is thus obtained:

$$
\frac{\mathrm{d} m}{\mathrm{~d} t} \sim \frac{-1}{\Delta \mathfrak{t}}\left[\frac{1-\gamma_{1}}{m+1}-\frac{1-\gamma_{1}}{s+1}\right] .
$$

This equation is valid only when $m<s$, or equivalently, $m<\left(N-m_{\mathrm{p}}\right) / 2$. It depicts the situation that the chain is pulled outward by the dominated entropic force from the outside. If $m$ is much smaller than $s$, the second term on the right-hand side of the equation can be ignored and the kinetics exhibit a scaling variation like

$$
\frac{\mathrm{d} m}{\mathrm{~d} t} \sim \frac{-1}{\Delta \mathfrak{t}} m^{-1} \sim \frac{-1}{\Delta \mathfrak{t}_{0}} m^{-z_{2 \mathrm{P}}} .
$$

It has been argued that the friction coefficient $\eta$ scales as $\eta_{0} m^{y_{2} \mathrm{P}}$ with $y_{2 \mathrm{P}}=2 \nu-1$ because the rested $m$ monomers in the cavity contribute to the energy dissipation at the non-confined stage [40]. As a result, the ejection speed shows $m^{-z_{2 \mathrm{P}}}$ variation with $z_{2 \mathrm{P}}=1+y_{2 \mathrm{P}}=2 \nu$.

Please notice that there exists an intermediate cavity size $D$ with the corresponding $N_{*}$ larger than $\left(N-m_{\mathrm{p}}\right) / 2$ but smaller than $N-m_{\mathrm{p}}$. In this situation, the system enters to the non-confined stage with the exterior chain length being still shorter than the interior one. As a consequence, a free energy barrier has to be surmounted first. The escape theory is applied again and the required escape time can be shown to be $\tau_{2 \mathrm{E}} \sim \frac{\eta_{0} \sigma^{2}}{k_{\mathrm{B}} T}\left[N^{2+x_{1}} I_{1}\left(\tilde{s}_{*}\right)+N^{2+y_{2 \mathrm{E}}} I_{2 \mathrm{E}}\left(\tilde{s}_{*}\right)\right]$ (cf. Appendix A in SI). After surmounting the free energy barrier, we have $s>m$ and the kinetics can be validly described by Eq. 5. The required time to pull the rest chain out of the cavity is $\tau_{2 \mathrm{P}} \sim \Delta \mathfrak{t}_{0} N_{*}^{1+z_{2 P}}$.

The final stage is the leaving stage which occurs when $s>N-m_{\mathrm{p}}$ and shows the free energy change $\Delta F(s)=\left(s-N+m_{\mathrm{p}}\right) \Delta \mu_{\mathrm{ps}}$. Here the chemical potential difference between the pore and the semi-space, $\Delta \mu_{\mathrm{ps}}$, is negative. The chain is confronted to a thermodynamic driving $\left|\Delta \mu_{\mathrm{ps}}\right| / \sigma$ and the leaving time is $\tau_{\text {leav }} \simeq m_{\mathrm{p}}\left(\frac{k_{\mathrm{B}} T}{\left|\Delta \mu_{\mathrm{ps}}\right|}\right) \Delta \mathfrak{t}_{0}$, which is generally short and negligible.

According to the physical pictures described above, the free energy landscape of an ejection process in the space of the state variables $s$ and $m$ is sketched in Figure 1. It can be 
classified into four situations, indicated by the roman numerals I to IV in the figure. Curve

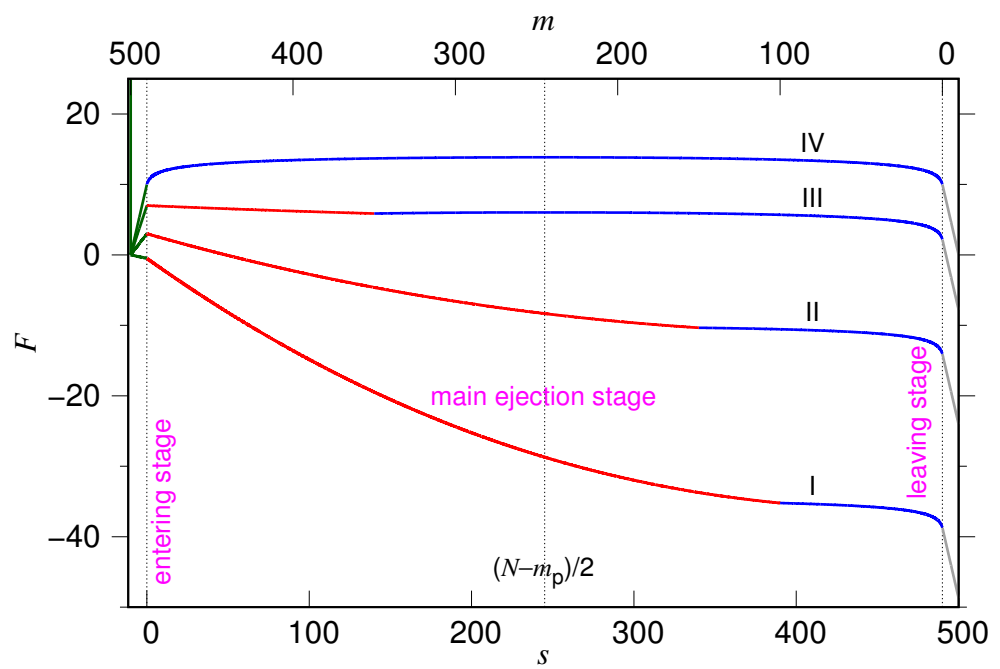

FIG. 1. Free energy $F$ as a function of the state variables $s$ (the bottom axis) and $m$ (the top axis). We choose $N=500$ and $m_{\mathrm{p}}=10$ to draw the sketch. In the entering and the leaving stages, the free energy curves are colored in dark-green and gray, respectively. In the main ejection stage, the curve is split into two sections: one in red color representing the confined stage and the other in blue color representing the non-confined stage. There exist four kinds of variations, denoted by I, II, III, and IV near the curves.

I presents the free energy change in an ejection process beginning with an initial volume fraction $\phi_{0}$ higher than the local volume fraction $\phi_{\mathrm{p}}$ of a monomer when it is presented in the pore. In this situation, the free energy decreases monotonically, from the entering stage, through the main ejection stage, to the leaving stage. The main ejection stage is subdivided into the confined and the non-confined stages, shown in red and blue colors, respectively. Curve II, similar to Curve I, depicts a large $\phi_{0}$ situation but the value of $\phi_{0}$ is smaller than $\phi_{\mathrm{p}}$. As a consequence, the free energy increases in the entering stage, which is a Kramers escape problem. The free energy then decreases monotonically in the following stages. The point demarcating the confined and the non-confined stages locates at $s=s_{*} \equiv N-m_{\mathrm{p}}-N_{*}$ which is right to the middle $\left(N-m_{\mathrm{p}}\right) / 2$. To meet the situation, the chain length must fulfill the condition $\left(N-m_{\mathrm{p}}\right)>2 N_{*}$. If the demarcating happens before reaching the middle of the process, the free energy does not monotonically decrease. There is a uphill to be mounted between $s=s_{*}$ and $s=\left(N-m_{\mathrm{p}}\right) / 2$. This is the situation III occurring when $N_{*}<\left(N-m_{\mathrm{p}}\right)<2 N_{*}$, where a second escape is required in the non-confined stage. If 
TABLE I. Predicted time scales $\tau_{1}, \tau_{2 \mathrm{E}}$, and $\tau_{2 \mathrm{P}}$ in the main ejection stage. Three situations are distinguished for the chain length: $N-m_{\mathrm{p}}<N_{*}, N_{*} \leq N-m_{\mathrm{p}} \leq 2 N_{*}$, and $N-m_{\mathrm{p}}>2 N_{*}$ where $N_{*} \sim(D / \sigma)^{1 / \nu}$ is the critical chain length. The ejection time $\tau_{\text {ej }}$ is a sum of the three time scales.

\begin{tabular}{|c|c|c|c|}
\hline \multirow{2}{*}{ chain length } & confined stage & \multicolumn{2}{|c|}{ non-confined stage } \\
\cline { 2 - 4 } & $\tau_{1} / \Delta \mathfrak{t}_{0}$ & $\tau_{2 \mathrm{E}} / \Delta \mathfrak{t}_{0}$ & $\tau_{2 \mathrm{P}} / \Delta \mathfrak{t}_{0}$ \\
\hline$N-m_{\mathrm{p}}<N_{*}$ & 0 & $N^{2+x_{1}} I_{1}(0)+N^{2+y_{2 \mathrm{E}}} I_{2 \mathrm{E}}(0)$ & $\frac{1}{1+z_{2 \mathrm{P}}}\left(\frac{N}{2}\right)^{1+z_{2 \mathrm{P}}}$ \\
$N_{*} \leq N-m_{\mathrm{p}} \leq 2 N_{*}$ & $\frac{N^{x_{1}}}{z_{1}-1} N_{*}^{2}\left[1-\left(\frac{N_{*}}{N}\right)^{z_{1}-1}\right]$ & $N^{2+x_{1}} I_{1}\left(\tilde{s}_{*}\right)+N^{2+y_{2 \mathrm{E}}} I_{2 \mathrm{E}}\left(\tilde{s}_{*}\right)$ & $\frac{1}{1+z_{2 \mathrm{P}}}\left(\frac{N}{2}\right)^{1+z_{2 \mathrm{P}}}$ \\
$N-m_{\mathrm{p}}>2 N_{*}$ & $\frac{N^{x_{1}}}{z_{1}-1} N_{*}^{2}\left[1-\left(\frac{N_{*}}{N}\right)^{z_{1}-1}\right]$ & 0 & $\frac{1}{1+z_{2 \mathrm{P}}} N_{*}^{1+z_{2 \mathrm{P}}}$ \\
\hline
\end{tabular}

$N-m_{\mathrm{p}}$ is smaller than $N_{*}$, the free energy looks similar to Curve IV. In this situation, the two escapes joint together, since the beginning at $s=-m_{\mathrm{p}}$ to the middle of the process at $s=\left(N-m_{\mathrm{p}}\right) / 2$. Once escaping the energy summit, the chain arrives the downhill side of the free energy for the rest of the process.

Based upon the above analysis, the non-confined stage can be distinguished into the thermal escape and the entropic pulling substages. The required time $\tau_{\text {ej }}$ for the main ejection stage is thus a sum of the three components: $\tau_{1}$ the time spent for the confined stage, $\tau_{2 \mathrm{E}}$ the time needed for the thermal escape substage, and $\tau_{2 \mathrm{P}}$ the one for the entropic

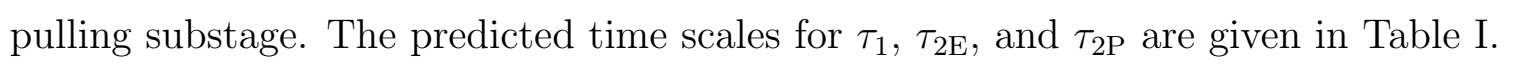

\section{SIMULATION MODEL AND SETTINGS}

In this work, we investigate the ejection process of a polymer in a quasi two-dimensional space and verify the scaling theory. We confine the polymer in a disklike cavity and study its ejection, through a small pore situated on the side of the cavity, into an outer slit space. The disklike cavity and the slit space are prepared by adding two parallel confining walls on top of the "spherical cavity-pore channel-semispace" system, as shown in Figure 2(a), which restricts the motion of polymer in a quasi two-dimensional space. The polymer is modeled by a bead-spring chain and loaded into the cavity by a pumping process. The loaded chain is then equilibrated with the head monomer being fixed at the pore entrance, which blocks the exit of the chain. The ejection is started by freeing the head monomer and the chain ejects spontaneously out of the cavity through the pore. The snapshots of the system are given in Figure 2(b) for the three processing phases: (i) loading, (ii) equilibration, and (iii) 
ejection.

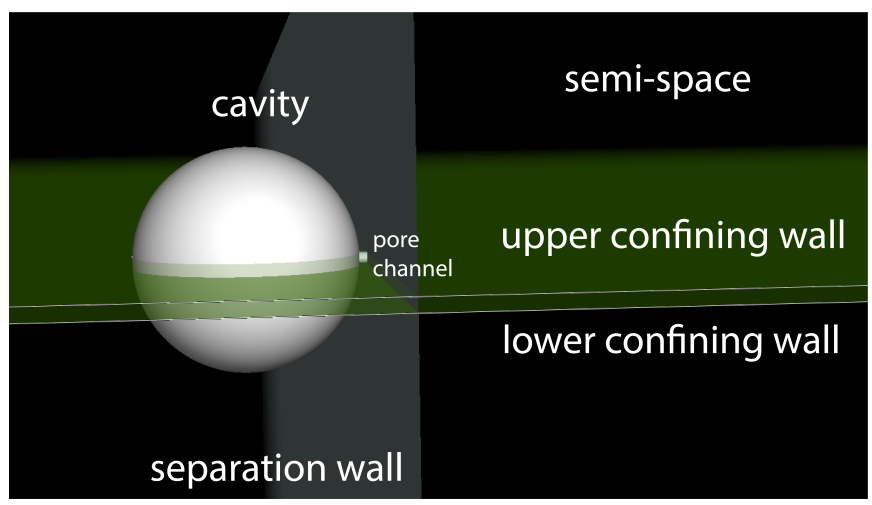

(a)
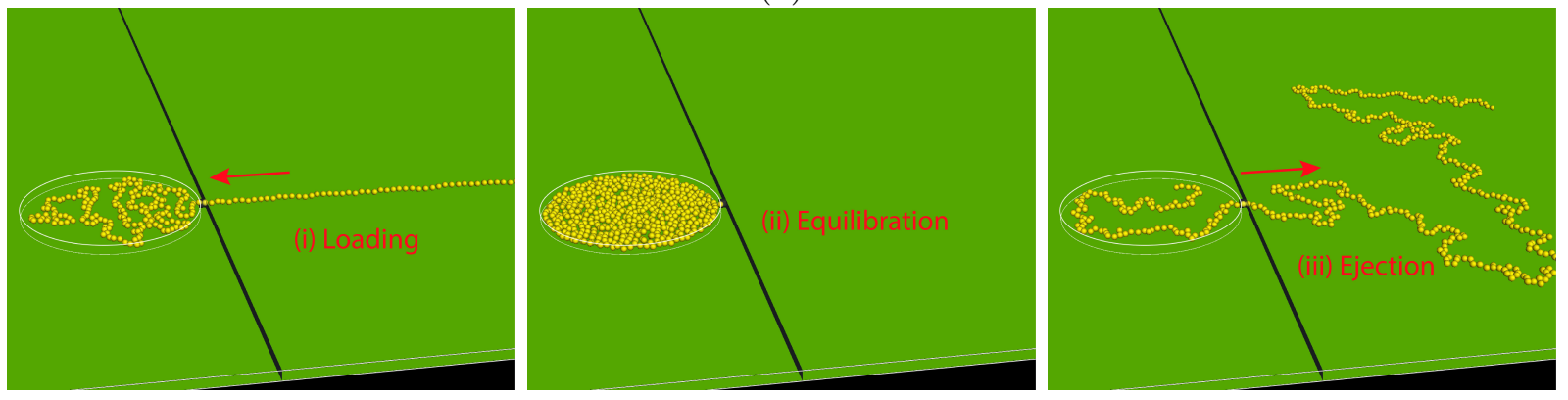

(b)

FIG. 2. (a) Setting of the quasi two-dimensional ejection system, which is realized by adding an upper and a lower confining wall on top of the three-dimensional "spherical cavity-pore channelsemispace" ejection system. (b) Snapshots of the system at the three processing phases: (i) loading, (ii) equilibration, and (iii) ejection. The chain has 512 beads and the volume fraction of beads after fully loaded into the cavity is 0.3 .

The chain beads interact with each others via a Lennard-Jones (LJ) 12-6 potential $U_{\text {ex }}(r)=4 \varepsilon\left[\left(\frac{\sigma}{r}\right)^{12}-\left(\frac{\sigma}{r}\right)^{6}\right]$, shifted and truncated at the minimum point $r_{\mathrm{c}}=\sqrt[6]{2} \sigma$, where $r$ is the distance between a pair of beads, and $\varepsilon$ and $\sigma$ are the interaction strength and length, respectively. The beads are connected by springs and form linear architecture. The potential of spring is $U_{\mathrm{sp}}(b)=\frac{1}{2} k\left(b-b_{0}\right)^{2}$ where $k$ is the spring constant and $b-b_{0}$ is the stretching length. The beads have repulsive interaction with the the confining wall via a LJ 9-3 potential, $U_{\mathrm{w}}(r)=\varepsilon_{\mathrm{w}}\left[\frac{2}{15}\left(\frac{\sigma_{\mathrm{w}}}{r}\right)^{9}-\left(\frac{\sigma_{\mathrm{w}}}{r}\right)^{3}\right]$, shifted and truncated at $r=\sqrt[6]{\frac{2}{5}} \sigma_{\mathrm{w}}$. We set $\varepsilon_{\mathrm{w}}=3.0 \varepsilon, \sigma_{\mathrm{w}}=\sigma, k=600 \varepsilon / \sigma^{2}$, and $b_{0}=\sigma$. The temperature $T$ is controlled by using Langevin thermostat [54] and set to be $1.0 \varepsilon / k_{\mathrm{B}}$. The relaxation time for the temperature 
control is $1.0 \sigma \sqrt{\mathfrak{m} / \varepsilon}$. Here $k_{\mathrm{B}}$ is the Boltzmann constant and $\mathfrak{m}$ is the mass of a bead. Under this setting, the wall potential attains the value of thermal energy $k_{\mathrm{B}} T$ at $r=0.76 \sigma$. It implies that the wall has an effective thickness equal to $0.26 \sigma$ because the bead radius is $0.5 \sigma$. Therefore, we set the cavity wall on a sphere of diameter $D_{\mathrm{C}}=D+0.52 \sigma$, which produces the desired disk diameter $D$ in the study. The two parallel walls are placed in a way to create a confining space with the gap height equal to $H=1.5 \sigma$. The pore channel is open on the side of the disklike cavity and modeled by a cylinder with the effective diameter $d_{\mathrm{p}}=1.5 \sigma$ and length $\ell_{\mathrm{p}}=1.0 \sigma$.

We vary the number of beads on a chain from $N=8$ to 1024 , as a power of 2 , and denote it by $N_{g_{N}}=2^{g_{N}}$. The diameter $D$ is varied to produce the desired initial volume fraction $\phi_{0}$ of monomers in the cavity at a given $N$. It can be shown that $\phi_{0}$ is related to $N, D$ and $H$ by

$$
\phi_{0}=N\left(\frac{\sigma}{D}\right)^{2} \times\left[\frac{2 \sigma}{3 H\left(1-\frac{1}{3}\left(\frac{H}{D}\right)^{2}\right)}\right]
$$

We see that $\phi_{0}$ is about equal to the two-dimensional volume fraction $N\left(\frac{\sigma}{D}\right)^{2}$ multiplying the factor $\frac{2 \sigma}{3 H}$ if $H \ll D$. We study the cases of the initial volume fraction equal to $\phi_{0, g_{F}}=$ $0.3 \times 2^{-g_{F}}$ where the generation number $g_{F}$ is varied from 0 to 10 . The corresponding diameter $D$ can be thus calculated by the formula

$$
D_{g_{D}}=\sigma \sqrt{\frac{2^{g_{D}}}{0.3}} \times \sqrt{\frac{2 \sigma}{3 H}+\frac{1}{3}\left(\frac{H}{\sigma}\right)^{2} \cdot 0.3 \times 2^{-g_{D}}} .
$$

Here $g_{D}=g_{N}+g_{F}$ is the generation number for $D$. Using the generation numbers allows us to investigate the scaling behaviors in a logical way. For each studied $\left(N_{g_{N}}, D_{g_{D}}, \phi_{0, g_{F}}\right)$ case, five hundred independent runs are performed. The simulation trajectories are recorded for post analysis. The case with $g_{F}=\infty$ is also studied for comparison. It simulates the situation that a chain is transported across a line barrier through a small pore in a quasi two-dimensional space. To prevent falling of the entire chain back into the cavity, especially when $D$ is large, we have set a reflective wall at the pore entrance which interacts only with the head monomer to bounce it back into the pore. Therefore, searching for the pore entrance from the inside of the cavity for the head monomer is not involved in this study.

In this paper, we choose $\mathfrak{m}, \sigma, \varepsilon$ to be the mass, length, energy units, respectively. A physical quantity will be reported only by its value in the following text, without explicitly 
mentioning the unit. For example, the ejection time " $\tau_{\text {ej }}=100.0$ " means $\tau_{\text {ej }}=100.0 t_{\mathrm{u}}$ where $t_{\mathrm{u}}=\sigma \sqrt{\mathfrak{m} / \varepsilon}$ is the time unit. The velocity " $V_{\mathrm{ej}}=3.0$ " means $V_{\mathrm{ej}}=3.0 \sigma / t_{\mathrm{u}}$.

\section{RESULTS}

\section{A. Ejection Speed}

The ejection kinetics is directly studied by calculating the averaged ejection speed $\left\langle V_{\mathrm{ej}}\right\rangle$. The speed is not a constant and varies a lot with time. As we will see, the largest ejection speed in a process could be hundred times, or even more, larger than the smallest speed, depending on the condition of ejection. Knowing the variation of $\left\langle V_{\mathrm{ej}}\right\rangle$ allows to understand how a chain is ejected at any moment of a process.

The speed $\left\langle V_{\mathrm{ej}}\right\rangle$ at a state $m$ is equal to the length of a segment, $\sigma$, dividing the averaged dwelling time $W(m)$ at the state. We recall that $m$ is the number of the monomers being still in the cavity. $W(m)$ is also called the waiting time function, which can be obtained from the simulations. Figure 3 presents the variation of $\left\langle V_{\mathrm{ej}}\right\rangle$ in an ejection process for different chain lengths and cavity diameters. Because $m$ decreases with time, the $\left\langle V_{\mathrm{ej}}\right\rangle$ curve should be looked from the right to the left in the figure to follow the direction of time. The chain length $N$ is varied from $N_{5}=32$ to $N_{10}=1024$ and the cavity diameter $D$ is varied from $D_{3}$ to $D_{18}$ in this study, where the values of $D_{g}$ are given by Eq. 7. The cases with infinite diameter $D=D_{\infty}$ are also studied for references, which corresponds to the translocation of polymer in a two-dimensional space.

We observe two main variational behaviors in a typical ejection process: $\left\langle V_{\mathrm{ej}}\right\rangle$ decreases at the confined stage and turns to show increasing behavior at the non-confined stage. On the $\log -\log$ plot, the two variations follows essentially the scaling $m^{z_{1}}$ and $m^{-z_{2 \mathrm{P}}}$, respectively, where the predicted exponents are $z_{1}=(d \nu-1)^{-1}=2.0$ and $z_{2 \mathrm{P}}=2 \nu=1.5$ by setting $d=2$ and $\nu=0.75$. The two values are significantly larger than the exponents for the three-dimensional ejection, $z_{1}=1.25$ and $z_{2 \mathrm{P}}=1.2$ [39, 40].

The kinetics equation in Eq. 2 implies that $\left\langle V_{\mathrm{ej}}\right\rangle$ should be identical to each other for different $N$ values at a given state $m$ if $D$ (and hence $N_{*}$ ) is fixed. However, the simulations reveal that the speed curve for a longer chain is evidently lower than the one for a shorter chain. To rectify it, we have introduced a chain length dependence for the friction coefficient, 


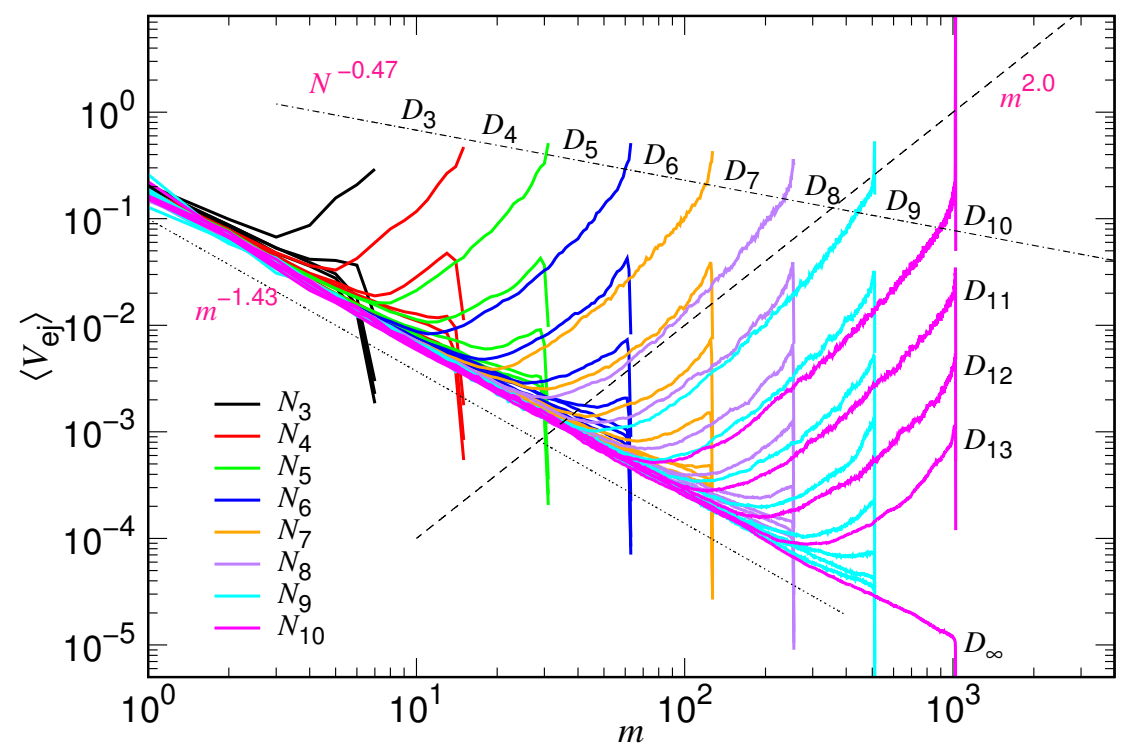

FIG. 3. Averaged ejection speed $\left\langle V_{\mathrm{ej}}\right\rangle$ as a function of the number of monomers $m$ in the cavity. The chain length $N$ is given in the legend by the formula $N_{i}=2^{i}$. The diameter $D$ of cavity is indicated on the top-right corner of the family curves which have the same $D_{g}$ value, calculated by using Eq. 7 .

$\eta \sim \eta_{0} N^{x_{1}}$, to reduce the ejection speed. The reason for the increase of $\eta$ with $N$ could be attributed to the effect of the geometrical restriction to rearrange the chain to be able to enter the pore channel and the impedance effect of the accumulated monomers near the pore exit in the semi-space. An exponent of $x_{1}=1 / 3$ has been found in the study of the three-dimensional ejection [39, 40]. Here we extend the scaling argument and anticipate that $x_{1}$ should be $1 / 2$ in our two-dimensional case. We can see that the ejection speed at starting (near $m=N$ ) descends with increasing the chain length as $\phi_{0}$ is fixed. It follows grosso modo a scaling $N^{-x_{1}}$, indicated by a dash-dotted line in the figure. In order to determine the value of $x_{1}$, we plot the rescaled speed $\left\langle V_{\mathrm{ej}}\right\rangle \times N^{x_{1}+z_{1}} m^{-z_{1}}$ in Figure 4 , by setting different values for $x_{1}$. Under the fixed- $\phi_{0}$ condition, the rescaled speed at the confined stage is expected to be constant for different chain lengths because

$$
V_{\mathrm{ej}} \sim \frac{m^{z_{1}}}{N^{x_{1}} N_{*}^{d \nu z_{1}}} v_{0} \sim \frac{m^{z_{1}}}{N^{x_{1}}(D / \sigma)^{d z_{1}}} v_{0} \sim \frac{m^{z_{1}}}{N^{x_{1}}\left(N / \phi_{0}\right)^{z_{1}}} v_{0}
$$

which is derived from Eq. 2 together with the scaling assumption for $\eta$. Here $v_{0}=\sigma / \Delta \mathfrak{t}_{0}$ is the characteristic speed, served as the unit.

As we can see, the rescaled curve is leveled off for a while as $m>N_{*}$ and becomes bent- 


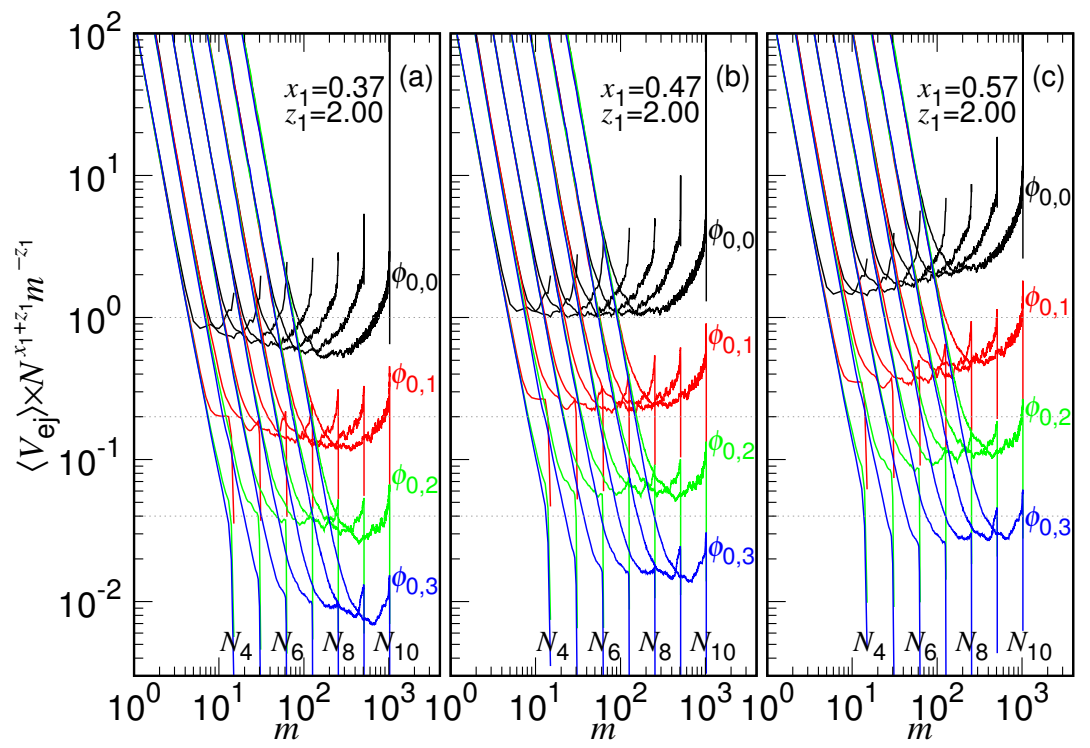

FIG. 4. Rescaled speed $\left\langle V_{\mathrm{ej}}\right\rangle \times N^{x_{1}+z_{1}} m^{-z_{1}}$ vs. $m$, by setting (a) $x_{1}=0.37$, (b) $x_{1}=0.47$, and (c) $x 1=0.57$. The exponent $z_{1}$ is assumed 2.0. The chain length $N_{i}=2^{i}$ is indicated near the bottom of the figure close to the curve. The initial volume fraction is indicated on the right side of the set of the curves sharing the same $\phi_{0}$ value, calculated by the formula $\phi_{0, j}=0.3 \times 2^{-j}$.

up as $m$ approaches $N$. The leveling-off value is found to be independent of $N$ by setting $x_{1}=0.47$ at the four studied $\phi_{0}$ values (refer to Panel (b)). Decreasing or increasing $x_{1}$ tilts the leveling to the right or the left, as shown in the panels (a) and (c) of the figure. It gives the best estimate for the $x_{1}$ exponent. We will come back to explain later why the curve is curved up near $m=N$.

If we plot $\left\langle V_{\mathrm{ej}}\right\rangle \times N^{x_{1}+z_{1}} m^{z_{2 \mathrm{P}}}$ versus $m$, the rescaled speed will be leveled off in the non-confined stage $\left(m<N_{*}\right)$, according to Eq. 5. The rescaling has still involved the factor $N^{x_{1}+z_{1}}$ in the multiplication, which guarantees the rescaled curves coinciding in the confined stage at a given $\phi_{0}$ value. Figure 5 shows the search for the value of the exponent $z_{2 \mathrm{P}}$. We find that setting $z_{2 \mathrm{P}}=1.43$ (see Panel $(\mathrm{b})$ ) produces the best overall horizontal lines in the non-confined stage. Decreasing or increasing $z_{2 \mathrm{P}}$ inclines the horizontal lines to the right (in Panel (a)) or to the left (in Panel (c)).

To understand the details of the speed scaling in the non-confined stage, we plot $\left\langle V_{\mathrm{ej}}\right\rangle$ as a function of $m$ for $D=\infty$ in Figure 6(a). We can see that the ejection increases its speed with decreasing $m$ and the speed curves follow a universal path when $m$ becomes small. According to the physical pictures described in Section III, the process stays entirely in a 


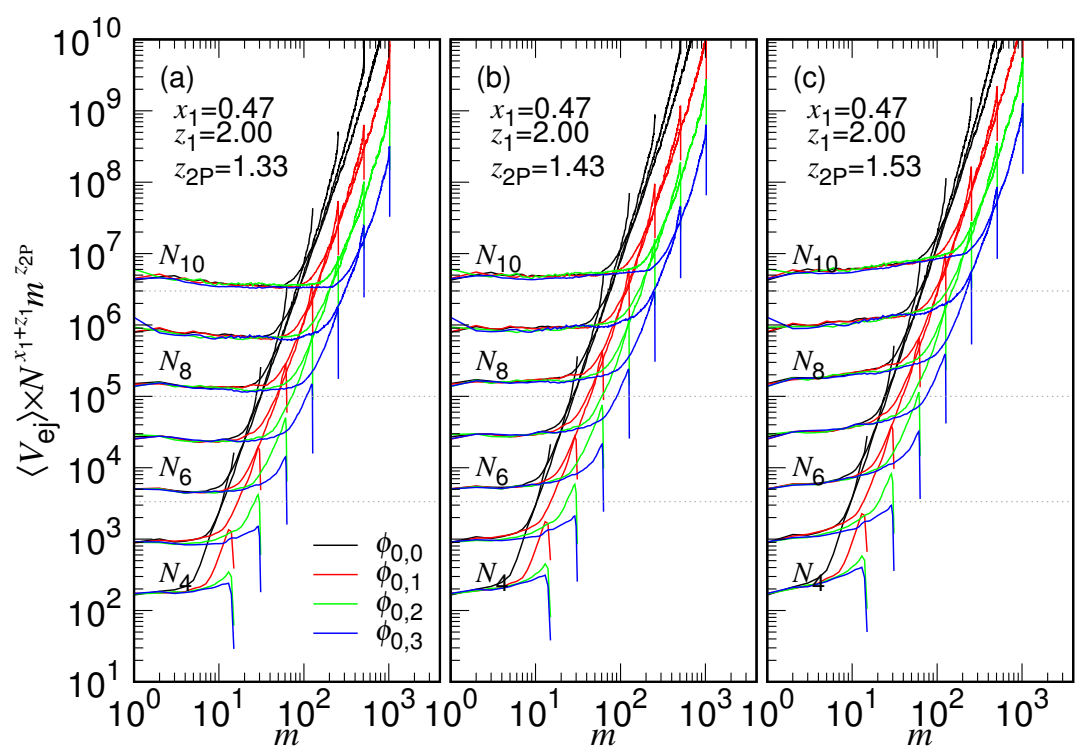

FIG. 5. $\left\langle V_{\mathrm{ej}}\right\rangle \times N^{x_{1}+z_{1}} m^{z_{2 \mathrm{P}}}$ versus $m$, by setting (a) $z_{2 \mathrm{P}}=1.33$, (b) $z_{2 \mathrm{P}}=1.43$, and (c) $z_{2 \mathrm{P}}=1.53$, with $x_{1}=0.47$ and $z_{1}=2.0$. The chain length $N_{i}=2^{i}$ is indicated on the left near the curve. The black, red, green, and blue curves have $\phi_{0}=\phi_{0, j}$ with $j=0,1,2,3$, respectively.

non-confined situation. In the first half part of the process $(m>0.5 N)$, the free energy of the system goes uphill and the "ejection" is a Kramers escape problem. We have derived a formula for the escape time (refer to the case $N-m_{\mathrm{p}}<N_{*}$ in Table II) and the ejection speed in this part exhibits an overall scaling with the exponent equal to $-z_{2 \mathrm{E}}$. In the second half part of the process, the number $m$ of the monomers in the cavity becomes smaller than the one $s$ in the semi-space. At this moment, the ejection is driven by the entropic pulling of the chain segments from the outside. The free energy goes downhill, and the ejection speed is described by Eq. 5 and scales as $V_{\mathrm{ej}} \sim m^{-z_{2 \mathrm{P}}}$. Figure 6(b) shows the variation of $\left\langle V_{\mathrm{ej}}\right\rangle$ vs. $m / N$. By performing non-linear regression fits in the two regions, $0.01<m / N<0.5$ and $0.5<m / N<0.95$, separately, for the chain lengths from $N=64$ to 1024 , we report the best estimates for the two exponents: $z_{2 \mathrm{P}}=1.43(3)$ and $z_{2 \mathrm{E}}=1.18(6)$. To see the difference of the exponents, we have plotted the rescaled speed $\left\langle V_{\mathrm{ej}}\right\rangle \times m^{1.43}$ versus $m / N$ in Figure 6(c). The rescaled curves are horizontal in the region $m / N<0.5$ and turn to show $m^{0.25}$ scaling when $m / N>0.5$

Despite the ejection speed can be well described by the kinetics equation Eq. 5 in the non-confined stage, a large bending-up effect, away from the predicted scaling $V_{\mathrm{ej}} \sim m^{z_{1}}$ for the confined stage, has been observed in Figure 3 particularly when the $m$ value is close to 


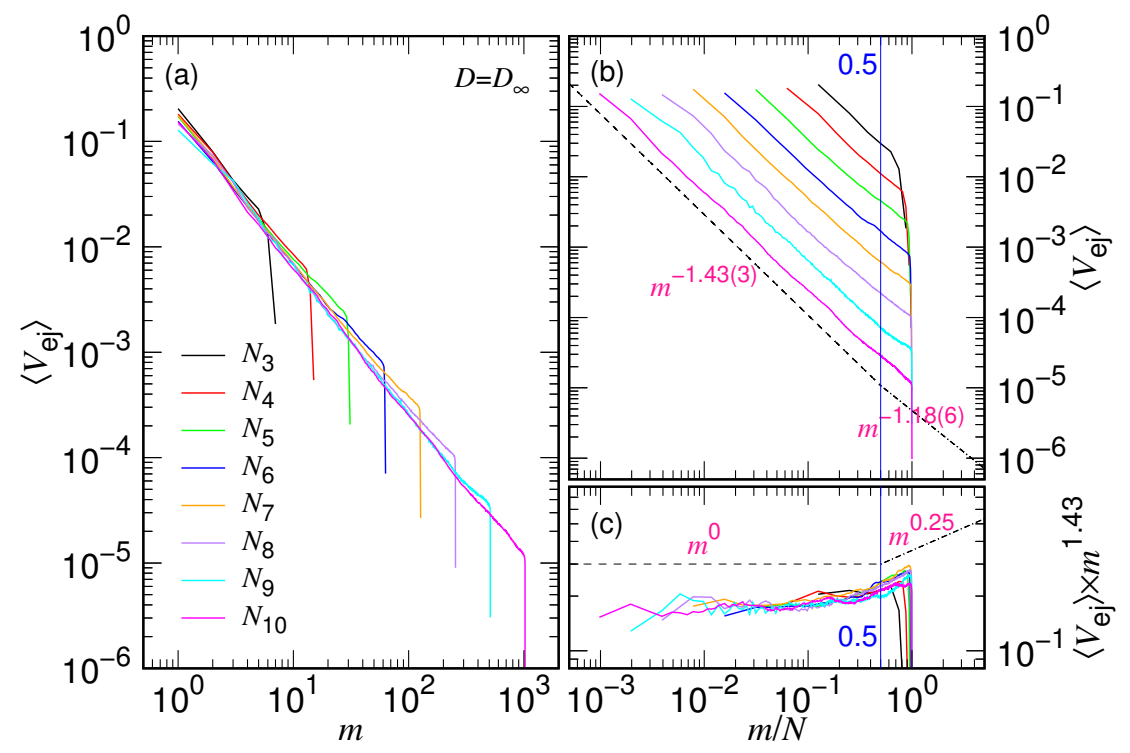

FIG. 6. (a) $\left\langle V_{\mathrm{ej}}\right\rangle$ vs. $m$, (b) $\left\langle V_{\mathrm{ej}}\right\rangle$ vs. $m / N$, and (c) $\left\langle V_{\mathrm{ej}}\right\rangle \times m^{1.43}$ vs. $m / N$, at $D=D_{\infty}$ for various chain lengths. The $N_{i}$ value is given by the formula $2^{i}$ and the color legend can be read in Panel (a).

$N$. The deviation occurs when the monomers are very dense in the cavity and the effect can be taken into account by considering the "second" virial term for the free energy [55]

$$
F \sim k_{\mathrm{B}} T\left[\left(\frac{m}{N_{*}}\right)^{z_{1}+1}+\frac{B_{2}}{2}\left(\frac{m}{N_{*}}\right)^{2\left(z_{1}+1\right)}\right]
$$

where $B_{2}$ is the 2 nd virial coefficient for the semidilute polymer solution. The kinetics equation for $m$ at the confined stage is thus rectified:

$$
\frac{\mathrm{d} m}{\mathrm{~d} t} \sim-\frac{z_{1}+1}{\Delta \mathfrak{t}_{0} N^{x_{1}}}\left(\frac{m^{z_{1}}}{N_{*}^{z_{1}+1}}\right)\left[1+B_{2}\left(\frac{m}{N_{*}}\right)^{z_{1}+1}\right] .
$$

The second virial term boosts the growing which bends the curve up in the log-log plot.

To verify the theory, we combine directly Eq. 10 and Eq. 5 and approximate the ejection speed for the whole process by using a single equation

$$
V_{\mathrm{ej}} \simeq v_{0}\left\{A_{1}\left(\frac{m^{z_{1}}}{N^{x_{1}}(D / \sigma)^{d z_{1}}}\right)\left[1+B_{2}\left(\frac{m^{z_{1}+1}}{(D / \sigma)^{d z_{1}}}\right)\right]+A_{2} m^{-z_{2 \mathrm{P}}}\right\}
$$

Here $A_{1}$ and $A_{2}$ are two prefactors which give the required weightings for the two scaling speeds derived separately in the confined and non-confined stages. We have utilized the scaling relation $D \sim \sigma N_{*}^{\nu}$ to substitute $N_{*}$ in the equation. Having the approximated speed 
equation on hand, we are able to perform non-linear regression fits for the simulation data and find $A_{1}=3.04, B_{2}=0.010$, and $A_{2}=0.20$. We make the plot of Eq. 11 in Figure 7(a) by plugging in the three fitting parameters for the set of the studied $N$ and $D$, using the theoretical exponents $x_{1}=0.5, z_{1}=2.0$, and $z_{2 \mathrm{P}}=1.5$.

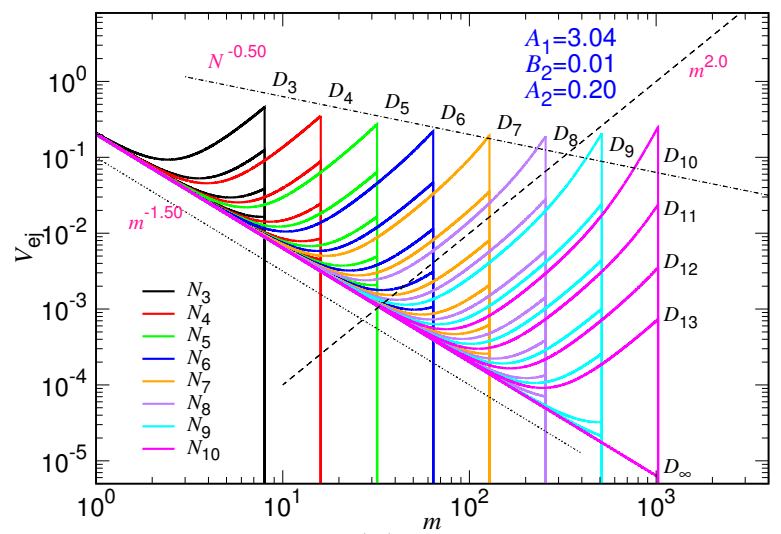

(a)

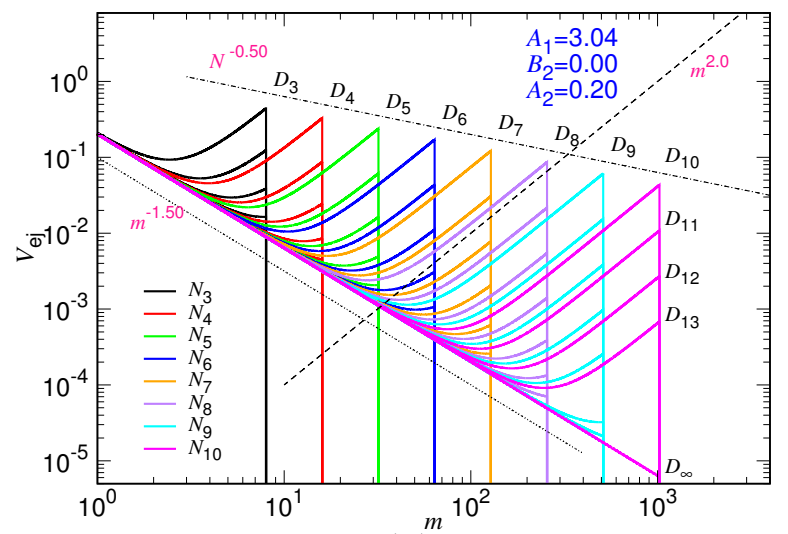

(b)

FIG. 7. Ejection speed $V_{\text {ej }}$ predicted by Eq. 11 for various $N$ and $D$ with the parameters: (a) $A_{1}=3.04, B_{2}=0.01, A_{2}=0.20$, and (b) $A_{1}=3.04, B_{2}=0, A_{2}=0.20$. The exponents $x_{1}, z_{1}$, and $z_{2 \mathrm{P}}$ are set to the two-dimensional values, $0.5,2.0$, and 1.5 , respectively. The chain length $N_{i}$ is $2^{i}$ and the cavity diameter $D_{g}$ is calculated by Eq. 7 .

Astonishing agreements in many features of the speed curves are observed between the theoretical figure and the simulations (Figure 3). For example, the ejection speed in the non-confined stage is well described by the scaling $m^{-z_{2 P}}$, while in the confined stage, the curve curves up from the scaling line $m^{z_{1}}$ as $m$ approaches $N$. The initial speed for different chain lengths does not well follow the predicted decreasing $N^{-x_{1}}$, as shown in Figure 3 . It is unlike the three-dimensional case [40] where the initial speed follows essentially $N^{-0.33}$. To see more clearly the impact of the second virial term on the two-dimensional ejection, we replot Eq. 11 by setting $B_{2}=0$ in Figure 7(b). We see right away that the two defectives are gone. The ejection speed in the confined stage and the initial speed both follow the desired scalings. It demonstrates the strong influence of the second virial term for the two-dimensional case, compared to the three-dimensional one.

Before ending this subsection, we study the scaling behavior of the critical number $N_{*}$. The number is determined by searching for the position of the minimum ejection speed in Figure 3. The results are given in Figure 8. 


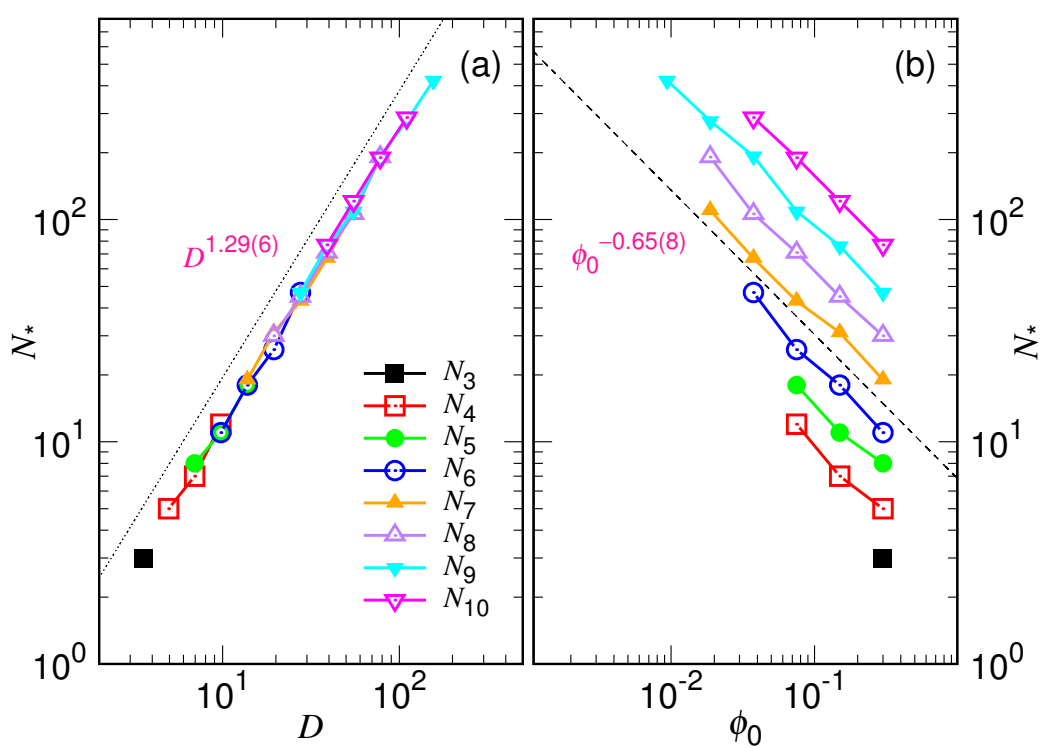

FIG. 8. (a) Critical monomer number $N^{*}$ as a function of the cavity diameter $D$ for various chain lengths $N$. (b) $N_{*}$ as a function of $\phi_{0}$ for different $N$. The color legend for $N$ is given in Panel (a) where $N_{i}=2^{i}$.

We find that $N_{*}$ scales as $D^{1.29(6)}$ in Panel (a), and is independent of $N$ because the data obtained from different chain lengths lie on a universal line. It agrees with our assumption that $D \sim \sigma N_{*}^{\nu}$, which implies $N_{*} \sim(D / \sigma)^{1 / \nu}$. Since $\nu$ is about 0.75 for a two-dimensional space, an exponent value around $4 / 3$ is expected on the figure. Concerning the scaling against the initial volume fraction, because $\phi_{0} \sim N(\sigma / D)^{d}$, we anticipate $N_{*} \sim\left(N / \phi_{0}\right)^{1 /(d \nu)}$. It gives $N_{*} \sim\left(N / \phi_{0}\right)^{2 / 3}$. This is exactly what we have observed in the panel (b). The sets of data decrease in a parallel manner for different chain lengths. The longer chain possesses a larger $N_{*}$ value at a given $\phi_{0}$.

\section{B. Decrease of Number of Monomers in the cavity}

We study the variation of the number of monomers $m$ in the cavity during an ejection process. Because the mean time $\langle t\rangle$ to reach a state $m$ depends on the chain length and the initial volume fraction, we rescale the time and the number of the monomers by the total time $\langle\tau\rangle$ and $N$, respectively, in order to compare different simulations. Figure 9(a) presents the results of $m / N$ vs. $\langle t\rangle /\langle\tau\rangle$ at three selected $\phi_{0}$ values for different chain lengths.

We observe that the number of the monomers does not decrease smoothly. A plateau 


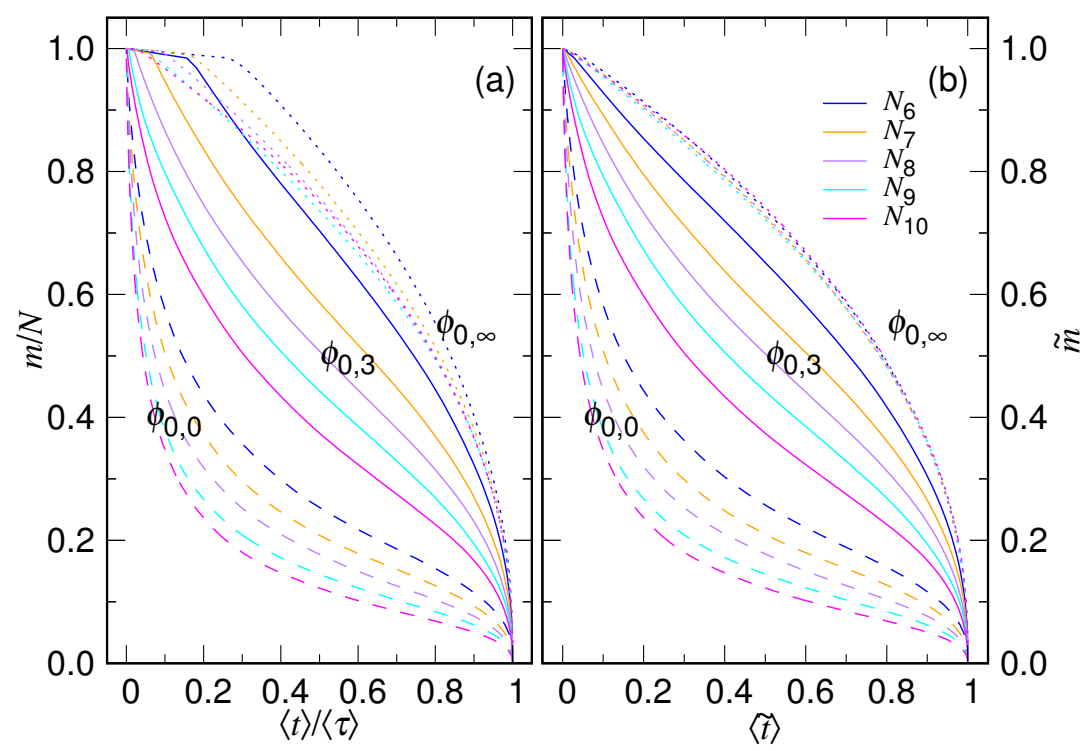

FIG. 9. (a) $m / N$ vs. $\langle t\rangle /\langle\tau\rangle$ and (b) $\tilde{m}$ vs. $\langle\tilde{t}\rangle$ at the three initial volume fractions: $\phi_{0,0} \equiv 0.3$ (in dashed line), $\phi_{0,3} \equiv 0.3 \times 2^{-3}$ (in solid line), and $\phi_{0, \infty} \equiv 0.0$ (in dotted line). The chain length can be read in the legend of Panel (b) where $N_{i} \equiv 2^{i}$.

region appears at the beginning of the curve, as $\phi_{0}$ is not large, with the value of $m$ descended by 1 . The descending number corresponds to the pore length $\ell_{\mathrm{p}}=1.0$. The phenomena have been identified in the three dimensional ejection and can be analogous to a nucleation process [39, 40]. Once the first monomer escapes the pore channel and reaches the semispace, the ejection can take place in a smooth way without stalling. Therefore, the required descending number 1 can be regarded as the "critical nucleus size" here for the further growth of the number of the monomers in the semi-space. At large $\phi_{0}$ value such as 0.3 , the plateau region does not appear and the curve decreases directly from the beginning.

To understand properly the decreasing behavior, we trim the critical nucleus size $m_{\mathrm{n}}$ off and replot the curves using the two trimmed variables: $\langle\tilde{t}\rangle=\left(\langle t\rangle-\tau_{\mathrm{n}}\right) /\left(\langle\tau\rangle-\tau_{\mathrm{n}}\right)$ and $\tilde{m}=\left(m-m_{\mathrm{n}}\right) /\left(N-m_{\mathrm{n}}\right)$ where $m_{\mathrm{n}}$ is one in this case and $\tau_{\mathrm{n}}$ is the the nucleation time which is equal to the time $\left\langle\tau_{\text {ent }}\right\rangle$ spent in the entering stage for the heading monomer to go across the pore. We can see in Figure 9(b) that the curves now decrease smoothly without stalling. At $\phi_{0}=\phi_{0, \infty}$, the trimmed curves are overlapped with each other, following a master descending curve for different chain lengths. With $\phi_{0}$ being increased, the concave curves turn to show covexness in the small $\langle\tilde{t}\rangle$ region. The longer the chain length, the faster the descending will be. 
The variation of $m$ with time can be predicted by solving the kinetics equation for the confined stage, Eq. 2, with the initial condition $m=N$ at $t=0$, and reads as

$$
\tilde{m} \simeq\left(1+\frac{t}{t_{0}}\right)^{-\zeta_{1}}
$$

where $t_{0} \sim \zeta_{1} N_{*}^{1+z_{1}} N^{1-z_{1}+x_{1}} \Delta \mathfrak{t}_{0}$ and $\zeta_{1}=\left(z_{1}-1\right)^{-1}$. For the non-confined stage, the variation can be solved from Eq. 5, with the "finial" state condition $m=0$ at $t=\tau_{\mathrm{ej}}$. We obtain

$$
\tilde{m} \simeq \frac{1}{N}\left(\frac{1}{\zeta_{2} \Delta \mathfrak{t}_{0}}\right)^{\zeta_{2}}\left(\tau_{\mathrm{ej}}-t\right)^{\zeta_{2}}
$$

where $\zeta_{2}=\left(z_{2}+1\right)^{-1}$. To understand how good the prediction is, we plot the two functions: $\left(\tilde{m}^{-1 / \zeta_{1}}-1\right) /\langle t\rangle$ vs. $m$ and $\tilde{m}$ vs. $\left\langle\tau_{\text {ej }}\right\rangle-\langle t\rangle$, from the simulations in Figure 10. The previous function is expected to be $1 / t_{0}$, which is constant for $m$ close to $N$ at large $\phi_{0}$. The latter should exhibit a power-law growth with an exponent $\zeta_{2}$ in the small $\left\langle\tau_{\mathrm{ej}}\right\rangle-\langle t\rangle$ region.

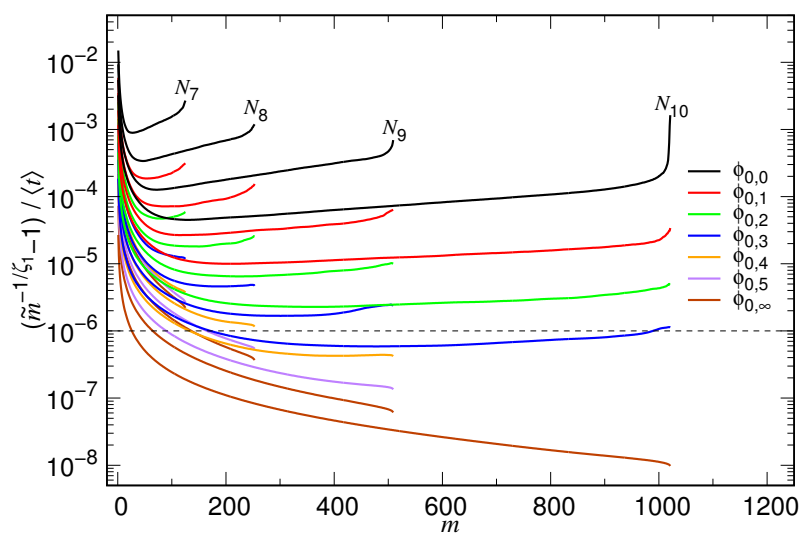

(a)

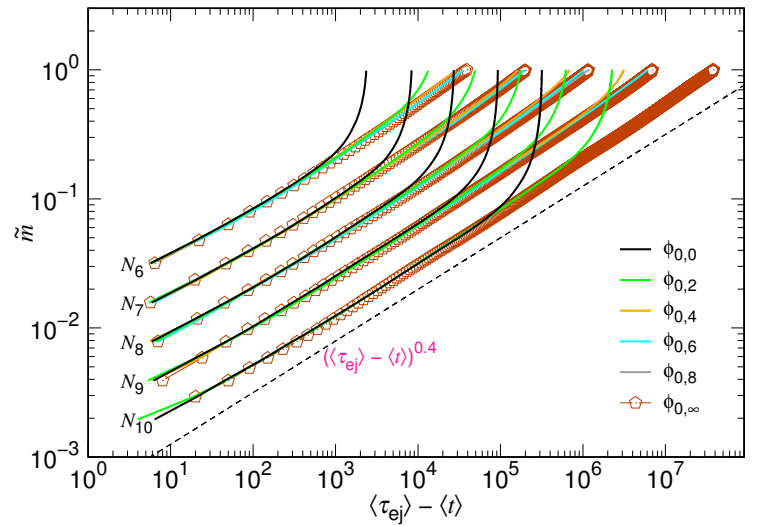

(b)

FIG. 10. (a) $\left(\tilde{m}^{-1 / \zeta_{1}}-1\right) /\langle t\rangle$ vs. $m$ by setting $\zeta_{1}=1.0$ and (b) $\tilde{m}$ vs. $\left\langle\tau_{\text {ej }}\right\rangle-\langle t\rangle$. The $\phi_{0}$ value can be read from the legends while the chain length $N$ is indicated directly near the corresponding curves.

Because the predicted $z_{1}$ exponent is 2.0 , we set $\zeta_{1}=1.0$ in the Panel (a) of the figure. We can see that the $\left(\tilde{m}^{-1 / \zeta_{1}}-1\right) /\langle t\rangle$ curves are essentially horizontal over a broad range of $m$ in the confined stage $\left(m<N_{*}\right)$ as $\phi_{0}$ is large for the chain length varied from $N_{7}$ to $N_{10}$. Figure 10(b) shows that $\tilde{m}$ follows a scaling relation $\left(\left\langle\tau_{\mathrm{ej}}\right\rangle-\langle t\rangle\right)^{0.4}$. The exponent $\zeta_{2}=0.4$ gives $z_{2}=1.5$, which corresponds well to the predicted value and the findings in Figure 3. The exponents $\zeta_{1}$ and $\zeta_{2}$ found here are significantly smaller than the ones for the three-dimensional ejection: $\zeta_{1}=4.0$ and $\zeta_{2}=0.454$ [40]. 
To understand the stalling for the decrease of $\langle m\rangle$ occurred at the beginning of the process, we performed simulations with $N=32$ for different pore lengths. The results are given in Figure 11(a) as a function of the normalized time $\langle t\rangle /\langle\tau\rangle$. The curves are plotted by using the "line-point" representation where each data point gives the required time to reach the given integer $m$ state.

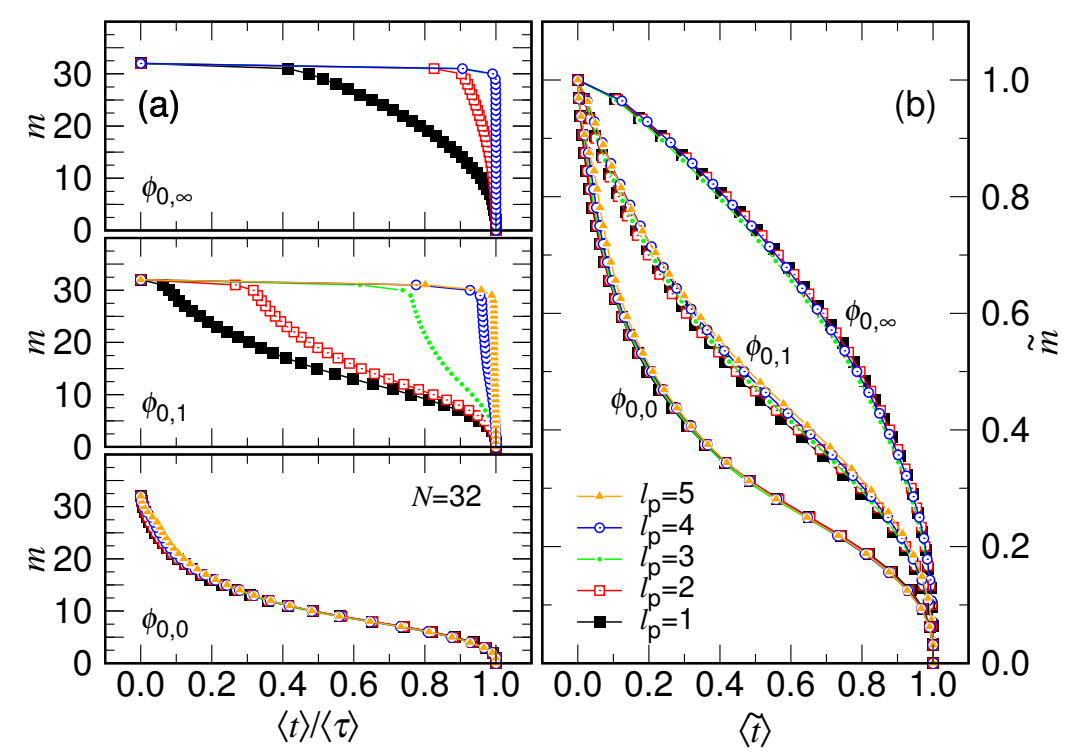

FIG. 11. (a) $m$ vs. $\langle t\rangle /\langle\tau\rangle$ for various pore length $\ell_{\mathrm{p}}$ at $\phi_{0}=\phi_{0, \infty}, \phi_{0,1}$, and $\phi_{0,0}$. (b) Time variation of the number of monomers plotted in the normalized coordinates $\tilde{m}$ and $\langle\tilde{t}\rangle$ at the three $\phi_{0}$. The value of $\ell_{\mathrm{p}}$ can be read in the legend.

The stalling time is found to increase with $\ell_{\mathrm{p}}$ at $\phi_{0}=\phi_{0,1}$. If we look carefully, we can see that the plateau constitutes exactly $m_{\mathrm{p}}$ data points. Similar behavior is also observed for $\phi_{0}<\phi_{0,1}$, for example, for the extreme case $\phi_{0}=\phi_{0, \infty}=0.0$ plotted at the top of the panel. The plateaus of the curves can be removed by shifting the time and the monomer number to be $\langle t\rangle-\tau_{\mathrm{n}}$ and $m-m_{\mathrm{n}}$, respectively, with the critical nucleus size $m_{\mathrm{n}}=m_{\mathrm{p}}$ and the nucleation time $\tau_{\mathrm{n}}=\left\langle\tau_{\text {ent }}\right\rangle$. The resulting curves exhibit astonishing overlaps on the normalized coordinates and become independent of $\ell_{\mathrm{p}}$, as given in Figure 11(b). It shows that the entering stage and the main ejection stage are decoupled. Each stage possesses its own scaling and can be studied independently. Please notice that at $\phi_{0}=\phi_{0,0} \equiv 0.3$, no plateau region appears on the curve. It is because the initial volume fraction of monomers in the cavity is about equal to the volume fraction $\phi_{\mathrm{p}}$ for a monomer to be presented in the channel [39, 40]. Here $\phi_{\mathrm{p}}$ can be evaluated by $\frac{1}{6} \pi \sigma^{3} /\left(\pi r_{\mathrm{p}}^{2} \sigma\right) \simeq 0.296$ with $r_{\mathrm{p}}=0.75 \sigma$ being 
the pore radius. In this case, we have set both $m_{\mathrm{n}}$ and $\tau_{\mathrm{n}}$ to zero.

Figure 12 (a) shows how the entering time varies with the pore length. In addition to $\phi_{0, g}=0.3 \times 2^{-g}$ with $g=0,1,2,3, \infty$, extra simulations have been performed at $\phi_{0}=0.2$, 0.25 and 0.4 to study the variation in details.

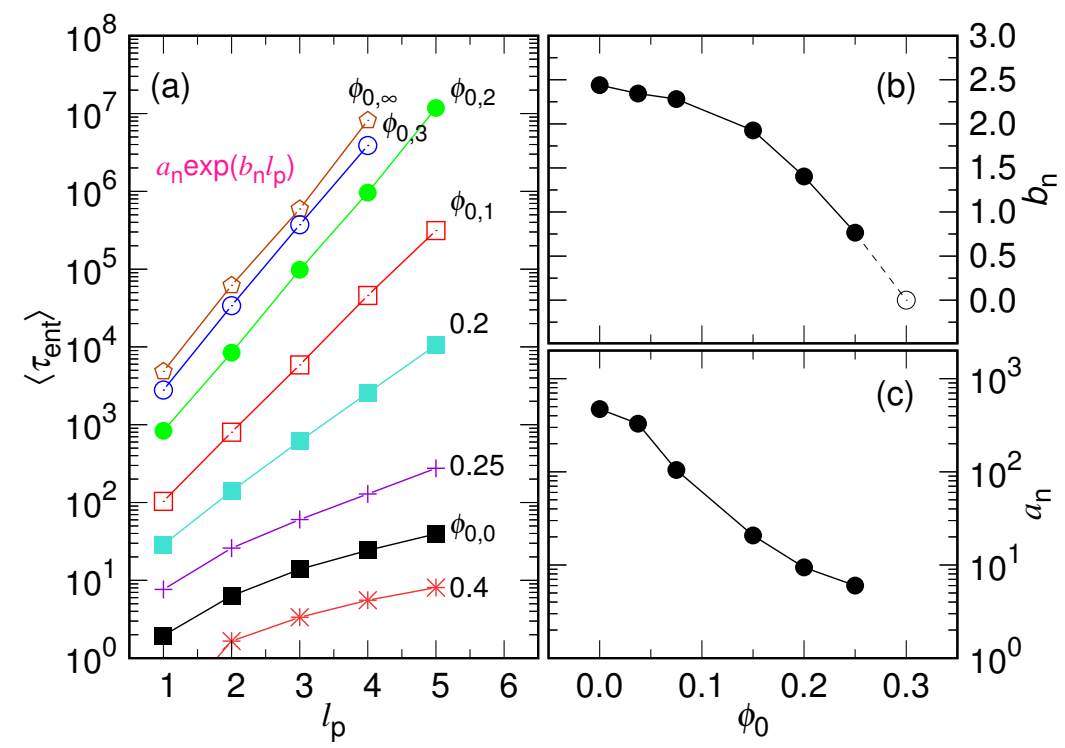

FIG. 12. (a) $\left\langle\tau_{\text {ent }}\right\rangle$ as a function of $\ell_{\mathrm{p}}$ in the semi-log plot. The value of $\phi_{0}$ is given near the corresponding curve. The data are fit by non-linear regression method via the form $a_{\mathrm{n}} \exp \left(b_{\mathrm{n}} \ell_{\mathrm{p}}\right)$ for $\phi_{0}>\phi_{\mathrm{p}}$. (b) $b_{\mathrm{n}}$ vs. $\phi_{0}$. (c) $a_{\mathrm{n}}$ vs. $\phi_{0}$.

We observe that the $\left\langle\tau_{\text {ent }}\right\rangle$ data falls on a straight line in the semi-log plot when $\phi_{0}$ is smaller than $\phi_{\mathrm{p}} \simeq 0.296$. It suggests that $\left\langle\tau_{\text {ent }}\right\rangle \simeq a_{\mathrm{n}} \exp \left(b_{\mathrm{n}} \ell_{\mathrm{p}}\right)$ with $a_{\mathrm{n}}$ and $b_{\mathrm{n}}$ being two parameters depending on $\phi_{0}$. For $\phi_{0} \geq \phi_{\mathrm{p}}$, the curve bends down in the semi-log plot and does not follow well the exponential growth. $b_{\mathrm{n}}$ and $a_{\mathrm{n}}$ can be obtained by nonlinear regression fits, and the results are presented in Panels (b) and (c). The value of $b_{\mathrm{n}}$ is about 2.5 at $\phi_{0}=0$ and decreases with increasing $\phi_{0}$. The extension of the $b_{\mathrm{n}}$ curve hits zero at $\phi_{0}$ around 0.3 , which corresponds to the $\phi_{\mathrm{p}}$ value. $a_{\mathrm{n}}$ decreases also with $\phi_{0}$. Different to $b_{\mathrm{n}}$, the magnitude reduces several orders.

The exponential dependence of $\left\langle\tau_{\text {ent }}\right\rangle$ on $\ell_{\mathrm{p}}$ suggests an Arrhenius type of transition for the heading monomers to go across the pore. An amount of energy $E_{\mathrm{a}}=m_{\mathrm{p}} \Delta \mu_{\mathrm{cp}}$ is required for the chain to gain from the thermal fluctuations to be able to send the first monomer to the semi-space for the following process. Here $m_{\mathrm{p}}=\ell_{\mathrm{p}} / \sigma$ is the number of monomers needed to span the pore and $\Delta \mu_{\mathrm{cp}}=\mu_{\mathrm{p}}-\mu_{\mathrm{c}}$ is the chemical potential difference between 
the cavity and the pore, which is directly related to the volume fraction difference between the two regions. $E_{\mathrm{a}}$ is thus the "activation energy", and the Arrhenius equation predicts the transition rate $k \propto \exp \left(-\frac{E_{\mathrm{a}}}{k_{\mathrm{B}} T}\right)$ which gives the required passage time across the pore to be about $\exp \left(\frac{\Delta \mu_{\mathrm{cp}}}{\sigma k_{\mathrm{B}} T} \ell_{\mathrm{p}}\right)$. A detailed calculation from the Fokker-Planck equation yields the same result: $\left\langle\tau_{\text {ent }}\right\rangle \sim \eta \exp \left(\frac{m_{\mathrm{p}} \Delta \mu_{\mathrm{cp}}}{k_{\mathrm{B}} T}\right)$, valid for $m_{\mathrm{p}} \Delta \mu_{\mathrm{cp}} \gg k_{\mathrm{B}} T$ (cf. Eq. S3 in Appendix A in SI). It is the "first passage time" of the Kramers' escape problem. We also call it the nucleation time here because the concept of nucleation has be adopted in the discussion.

If $m_{\mathrm{p}} \Delta \mu_{\mathrm{cp}}$ is not much greater than $k_{\mathrm{B}} T$, Eq. S3 predicts $\left\langle\tau_{\text {ent }}\right\rangle \sim \frac{\eta \ell_{\mathrm{p}}^{2}}{2 k_{\mathrm{B}} T}$ for $m_{\mathrm{p}}\left|\Delta \mu_{\mathrm{cp}}\right| \ll$ $k_{\mathrm{B}} T$, happened when $\phi_{0}$ is close $\phi_{\mathrm{p}}$, and $\left\langle\tau_{\mathrm{ent}}\right\rangle \sim\left(\frac{\eta \sigma}{\left|\Delta \mu_{\mathrm{cp}}\right|}\right) \ell_{\mathrm{p}}$ for $m_{\mathrm{p}} \Delta \mu_{\mathrm{cp}}<-k_{\mathrm{B}} T$, happened when $\phi_{0} \gg \phi_{\mathrm{p}}$. The previous situation predicts a diffusion-like motion for the head monomer to come across the pore and therefore, $\left\langle\tau_{\text {ent }}\right\rangle$ is proportional to $\ell_{\mathrm{p}}^{2}$. The latter depicts a constant motion where the heading monomers are driven through the pore by a driving force $\frac{\left|\Delta \mu_{\mathrm{cp}}\right|}{\sigma}$, which produces a mean drift speed $\frac{\left|\Delta \mu_{\mathrm{cp}}\right|}{\eta \sigma}$. Consequently, $\left\langle\tau_{\mathrm{ent}}\right\rangle$ is proportional to $\ell_{\mathrm{p}}$. The data for $\phi_{0}=0.3$ and 0.4 in Panel (a) correspond to the two situations and hence, do not lie on a straight line in the semi-log plot.

To make evident the scaling, we replot $\left\langle\tau_{\text {ent }}\right\rangle$ against $\ell_{\mathrm{p}}$ in Figure 13(a) using the log-log scales.

We can see that $\left\langle\tau_{\text {ent }}\right\rangle$ behaves as $\ell_{\mathrm{p}}^{2.04(5)}$ at $\phi_{0}=0.3$. The $\phi_{0}$ value is about $\phi_{\mathrm{p}}$ and therefore corresponds to the case showing the diffusive kinetics. Increasing $\phi_{0}$ to 0.4 gives $\left\langle\tau_{\text {ent }}\right\rangle \sim \ell_{\mathrm{p}}^{1.72(6)}$. It exhibits a super-diffusion behavior with the diffusion exponent equal to $2 / 1.72=1.16$. The $\phi_{0}$ value is not high enough to show pure constant motion in the entering stage with $\left\langle\tau_{\text {ent }}\right\rangle \propto \ell_{\mathrm{p}}$. We cannot go further to investigate higher- $\phi_{0}$ cases, because the volume fraction has been close to the close pack value in our quasi-2D confining model. It is known that the packing fraction of a close pack in a pure two-dimensional space is $\phi_{\mathrm{cl}}^{(2 \mathrm{D})}=\frac{\pi r^{2} / 2}{\sqrt{3} r^{2}}=0.9069$. In our model, the volume fraction is related to the corresponding $2 \mathrm{D}$ packing by $\phi_{0}=\phi_{0}^{(2 \mathrm{D})} \times \frac{2 \sigma}{3 H}$. A close-pack $2 \mathrm{D}$ arrangement thus gives $\phi_{0} \simeq 0.4030$ because $H=1.5 \sigma$ in this study. The value $\phi_{0}=0.4$ has been about the maximum volume fraction that we can investigate. For the cases with $\phi_{0}$ smaller than 0.3 , the data follow the exponential growth with $\ell_{\mathrm{p}}$, as shown in the figure.

Figure 13(b) compares the descending behavior of $m$ for $\ell_{\mathrm{p}}=5$ at various $\phi_{0}$ values. Figure 13 (c) is the zoom-in of the curves near the starting point, $m=32$. We can see that the descending behavior, and thus the kinetics, changes when $m$ goes across the number 


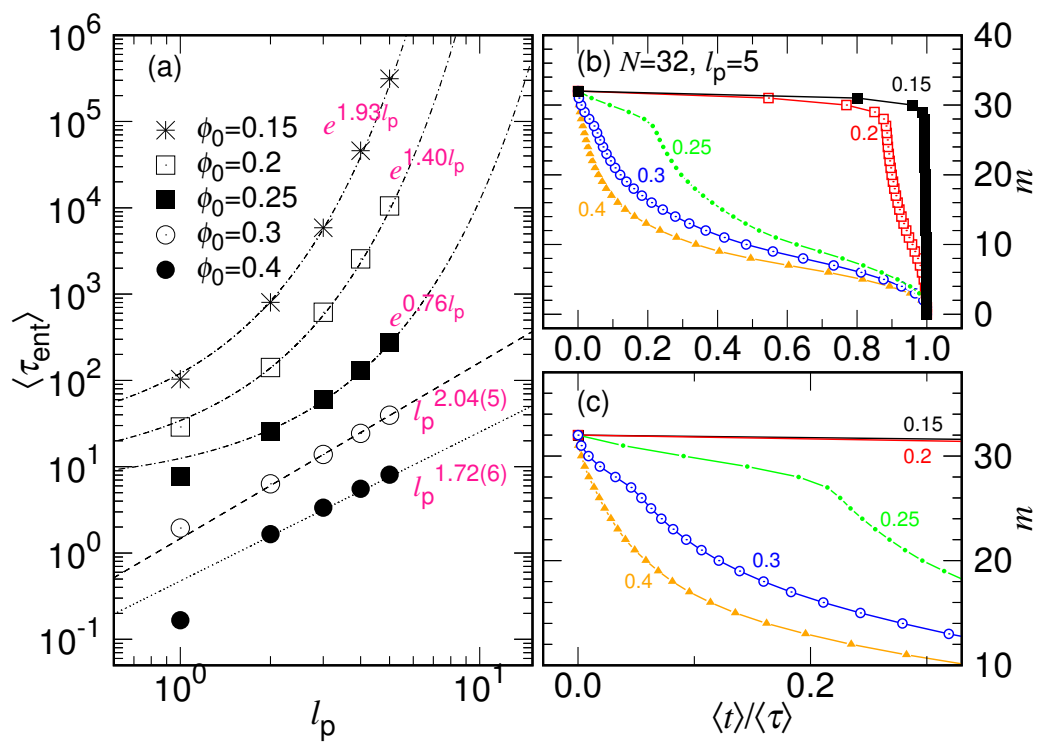

FIG. 13. (a) $\left\langle\tau_{\text {ent }}\right\rangle$ as a function of $\ell_{\mathrm{p}}$ in $\log -\log$ scales. The chain length is 32 and the value of $\phi_{0}$ is indicated in the legend. (b) Descending of $m$ plotted in the normalized time coordinate $\langle t\rangle /\langle\tau\rangle$. The pore length $\ell_{\mathrm{p}}$ is 5.0. The $\phi_{0}$ value is given near the corresponding curve. Panel (c) is a zoom-in of the panel (b) near the starting point $m=32$.

$N-m_{\mathrm{p}}=27$. For $\phi_{0}<\phi_{\mathrm{p}}$, the descending curve is concave in the entering stage. It changes to show convexness when $\phi_{0}$ becomes close or larger than $\phi_{\mathrm{p}}$.

\section{Processing Time Analysis}

The scaling behavior of the spending time in an ejection process is analyzed in this section. The process can be divided into the three stages: (1) the entering stage, for the head monomer to enter and pass through the pore channel, (2) the main ejection stage, for the body of the chain to be transported from the disklike cavity, through the channel, to the semi-space, and (3) the leaving stage, for the tail monomer to leave the pore channel to the semi-space. The total processing time is thus the sum of the duration in the three stages, $\langle\tau\rangle=\left\langle\tau_{\text {ent }}\right\rangle+\left\langle\tau_{\text {ej }}\right\rangle+\left\langle\tau_{\text {leav }}\right\rangle$. The main ejection stage can be further separated into the two stages: the confined stage, in which the chain segments suffer from the confinement of the cavity and are pressed out of it, and the non-confined stage, occurred when the pervaded space of the rested chain segments becomes smaller than the cavity size. The ejection time is thus equal to $\left\langle\tau_{\mathrm{ej}}\right\rangle=\left\langle\tau_{1}\right\rangle+\left\langle\tau_{2}\right\rangle$, the sum of the time in the two stages. We have argued 
that $\left\langle\tau_{2}\right\rangle$ for the non-confined stage can be furthermore split into the two terms, $\left\langle\tau_{2 \mathrm{E}}\right\rangle$ and $\left\langle\tau_{2 \mathrm{P}}\right\rangle$, according to the way of the chain being driven. $\left\langle\tau_{2 \mathrm{E}}\right\rangle$ is the thermal escape time occurred when the chain length in the cavity is still longer than the one in the semi-space in the non-confined stage, while $\left\langle\tau_{2 \mathrm{P}}\right\rangle$ is the time spent for the chain driven by the entropic pulling from the outside when the outer chain becomes longer than the remaining one in the cavity. A sketch for the free energy change in these stages has been given in Figure 1.

We first study the variations of the decomposed time as a function of $N$, in Figure 14 . under the fixing $D$ and fixing $\phi_{0}$ conditions. Panel (a) reveals that at a given $D,\left\langle\tau_{\text {ent }}\right\rangle$

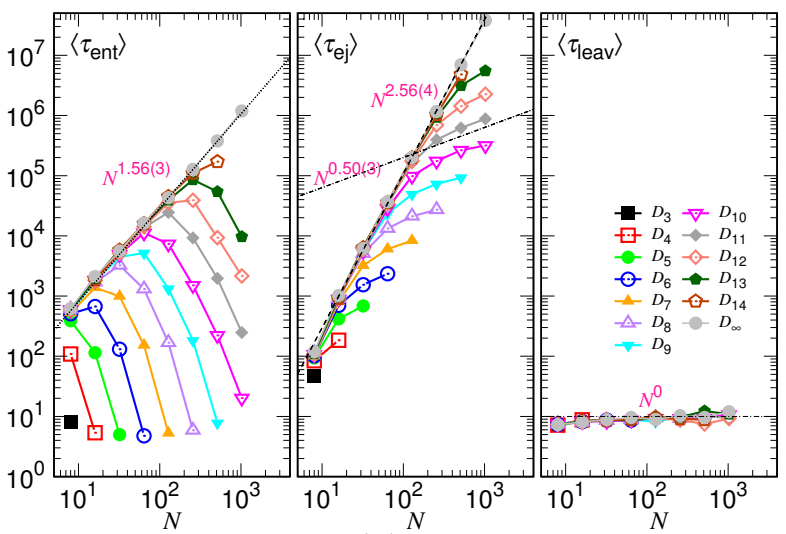

(a)

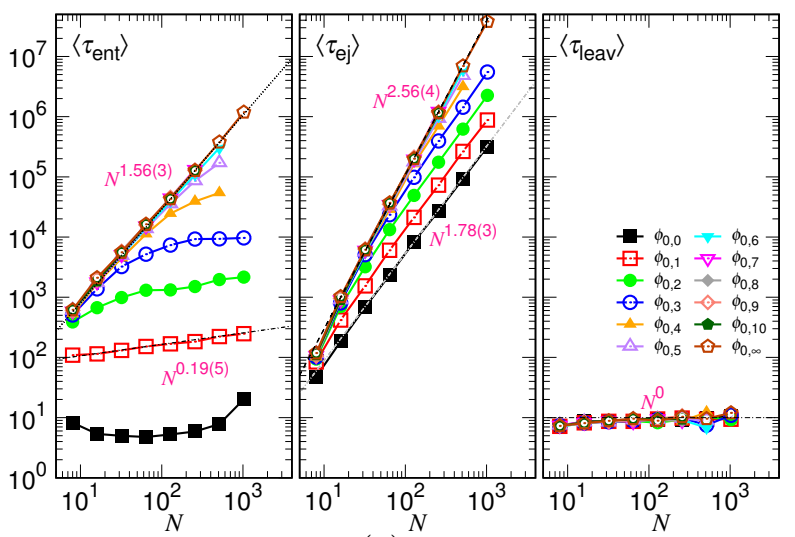

(c)

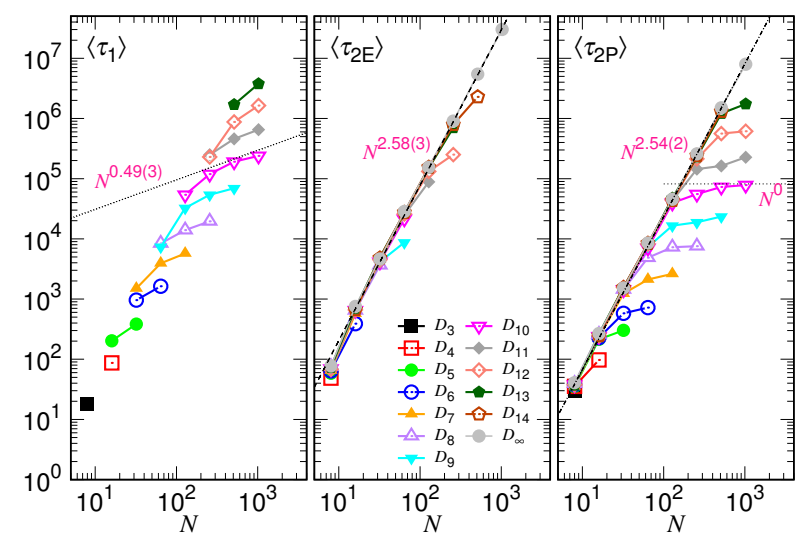

(b)

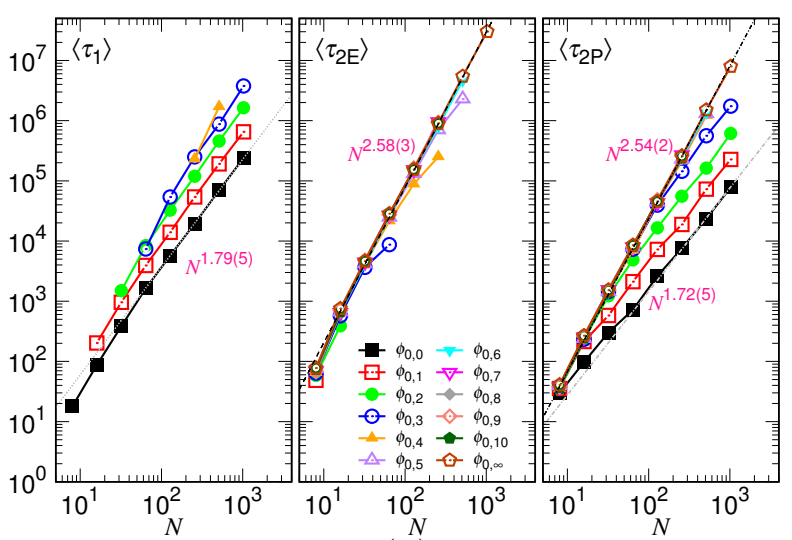

(d)

FIG. 14. (a) $\left\langle\tau_{\text {ent }}\right\rangle,\left\langle\tau_{\text {ej }}\right\rangle,\left\langle\tau_{\text {leav }}\right\rangle$, and (b) $\left\langle\tau_{1}\right\rangle,\left\langle\tau_{2 \mathrm{E}}\right\rangle,\left\langle\tau_{2 \mathrm{P}}\right\rangle$ as a function of $N$, plotted under the $D$-fixed condition. The value of $D$ can be read in the legends, calculated by Eq. 7. (c) $\left\langle\tau_{\text {ent }}\right\rangle,\left\langle\tau_{\text {ej }}\right\rangle$, $\left\langle\tau_{\text {leav }}\right\rangle$, and (d) $\left\langle\tau_{1}\right\rangle,\left\langle\tau_{2 \mathrm{E}}\right\rangle,\left\langle\tau_{2 \mathrm{P}}\right\rangle$ as a function of $N$, plotted under the $\phi_{0}$-fixed condition. The value of $\phi_{0}$ can be read in the legends, calculated by $\phi_{0, j}=0.3 \times 2^{-j}$.

grows like $N^{1.56(3)}$ and turns to show fast decreasing behavior as $N$ becomes larger than the 
critical $N_{*} \sim(D / \sigma)^{1 / \nu}$. If it is $\phi_{0}$ being fixed (see Panel $\left.(\mathrm{c})\right),\left\langle\tau_{\text {ent }}\right\rangle$ exhibits a change of the scaling behavior from $N^{0.19(5)}$ to $N^{1.56(3)}$ with decreasing the $\phi_{0}$ value. Recall that $\left\langle\tau_{\text {ent }}\right\rangle$ is the nucleation time and varies as $\eta \exp \left(\frac{\ell_{\mathrm{p}} \Delta \mu_{\mathrm{cp}}}{\sigma k_{\mathrm{B}} T}\right)$. The fixed- $\phi_{0}$ study implies that $\Delta \mu_{\mathrm{cp}}$ is fixed because the chemical potential difference is directly related to the volume fraction difference between the cavity and the pore. It is thus the change of the effective friction coefficient $\eta$ which leads to the change of the scaling. In the previous three-dimensional study [39, 40], we have obtained the nucleation time be to $N^{0.32(2)}$ at large $\phi_{0}$ and $N^{1.58(6)}$ as $\phi_{0}$ becomes small. At that study, we have proposed a scaling argument for the change of $\eta$ from $N^{x_{1}}$ to $N^{1+\nu}$ with decreasing $\phi_{0}$. It looks that the proposed scaling fails to be extended for the two-dimensional case. Therefore, the mechanism which influences the change of the effective friction in the entering (nucleation) stage requires further exploration in the future.

The ejection time $\left\langle\tau_{\mathrm{ej}}\right\rangle$ changes from $N^{2.56(4)}$ to $N^{0.50(3)}$ with increasing $N$ in Panel (a). The results are very close to the prediction given in Table If $\tau_{\mathrm{ej}}$ is dominated by $\tau_{2}$, scaling like $N^{1+z_{2 \mathrm{P}}}$ when $N<N_{*}$, and by $\tau_{1}$, scaling like $N^{x_{1}} N_{*}^{2}$ when $N$ is much larger than $N_{*}$, where the expected $z_{2 \mathrm{P}}$ and $x_{1}$ exponents are $2 \nu$ and $1 / d$, respectively, with $\nu=\frac{3}{d+2}=0.75$ in the two-dimensional $(d=2)$ space. If the $\phi_{0}$-fixed condition is applied, the scaling is switched from $N^{1.78(3)}$ to $N^{2.56(4)}$ by reducing the $\phi_{0}$ value, as seen in Panel (c). The two exponents agree with the predictions $x_{1}+\frac{2}{d \nu} \simeq 1.83$ and $1+z_{2 \mathrm{P}}=2.5$, respectively. Here the additional term $\frac{2}{d \nu}$ comes into the first exponent because the dominated time $\tau_{1}$ has the extra scaling dependence $N_{*}^{2} \sim\left(N / \phi_{0}\right)^{2 /(d \nu)}$ on $N$ as $\phi_{0}$ is fixed.

Panels (a) and (c) also show that the leaving time $\left\langle\tau_{\text {leav }}\right\rangle$ is negligibly small in comparison with $\left\langle\tau_{\text {ent }}\right\rangle$ and $\left\langle\tau_{\mathrm{ej}}\right\rangle$, and is basically a constant.

The decomposition of $\left\langle\tau_{\mathrm{ej}}\right\rangle$ shows that $\left\langle\tau_{1}\right\rangle$ scales like $N^{0.49(3)}$ in Panel (b) and $N^{1.79(5)}$ in Panel (d). They are close the predicted scaling $N^{x_{1}}$ and $N^{x_{1}+(2 / d \nu)}$ for the $D$-fixed and $\phi_{0}$-fixed cases. The $\left\langle\tau_{2 \mathrm{E}}\right\rangle$ data in the two panels lie basically on a line $N^{2.58(3)}$. It has been shown in Figure 6 that $z_{2 \mathrm{E}}$ is about 1.18, which gives $y_{2 \mathrm{E}} \simeq 0.18$ smaller than $x_{1}$. The dominated scaling for $\left\langle\tau_{2 \mathrm{E}}\right\rangle$ is thus $N^{2+x_{1}}$ according to Table I. The $\left\langle\tau_{2 \mathrm{P}}\right\rangle$ time is expected to show $N_{*}^{1+z_{2 \mathrm{P}}}$ for $N>2 N_{*}$ and $N^{1+z_{2 \mathrm{P}}}$ for $N<2 N_{*}$. Again, the obtained exponents, 1.72(5) at the large $\phi_{0}$ and 2.54(2) at the small $\phi_{0}$ in Panel (d), are quite close to the theoretical values $\frac{1+z_{2 \mathrm{P}}}{d \nu} \simeq 1.67$ and $1+z_{2 \mathrm{P}}=2.5$, respectively.

The variations of the different time components versus $D$ for the $\phi_{0}$-fixed and $N$-fixed conditions are given in Figure 15. We see in Panel (a) that $\left\langle\tau_{\text {ent }}\right\rangle$ scales as $D^{0.36(4)}$ at 


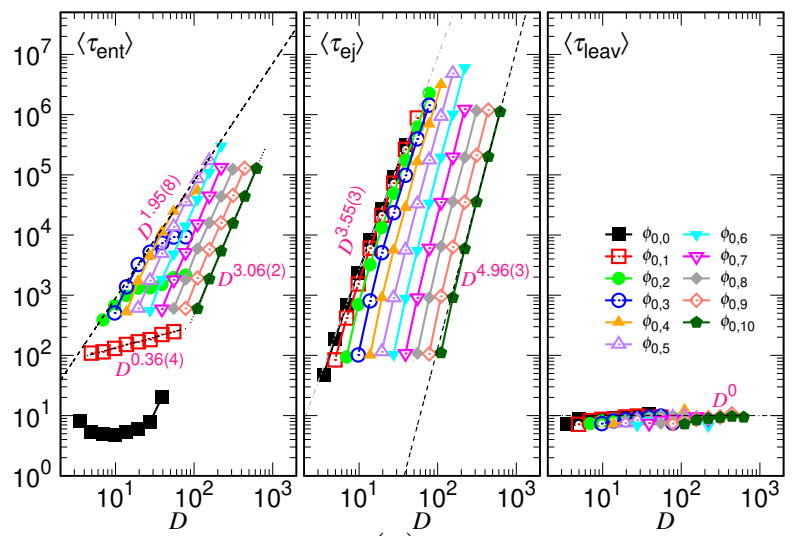

(a)

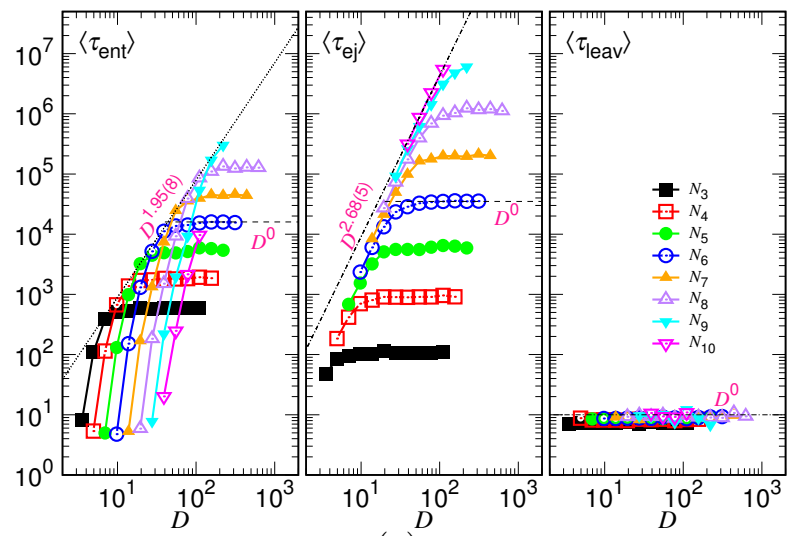

(c)

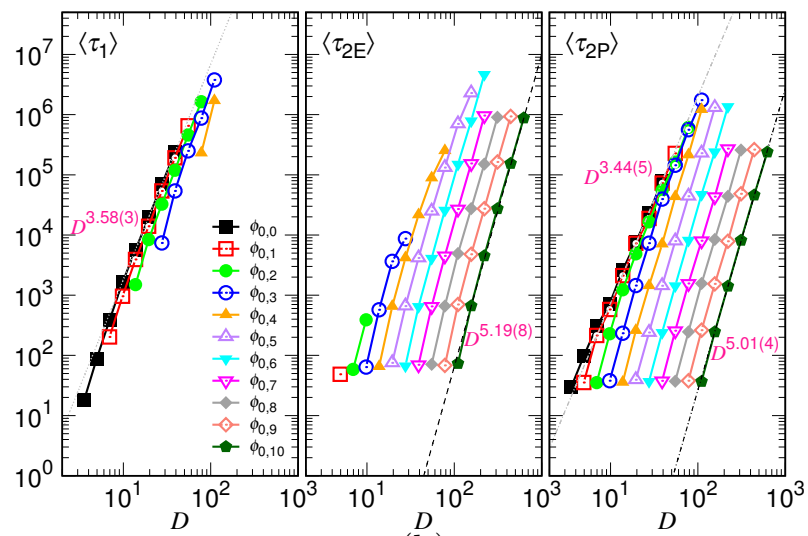

(b)

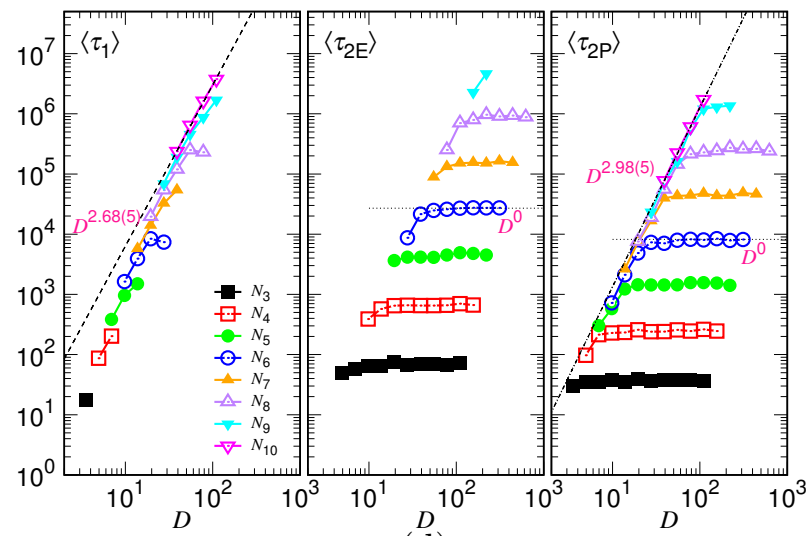

(d)

FIG. 15. (a) $\left\langle\tau_{\text {ent }}\right\rangle,\left\langle\tau_{\text {ej }}\right\rangle,\left\langle\tau_{\text {leav }}\right\rangle$, and (b) $\left\langle\tau_{1}\right\rangle,\left\langle\tau_{2 \mathrm{E}}\right\rangle,\left\langle\tau_{2 \mathrm{P}}\right\rangle$ as a function of $D$, plotted under the $\phi_{0}$-fixed condition. The value of $\phi_{0}$ can be read in the legends, calculated by $\phi_{0, j}=0.3 \times 2^{-j}$. (c) $\left\langle\tau_{\text {ent }}\right\rangle,\left\langle\tau_{\text {ej }}\right\rangle,\left\langle\tau_{\text {leav }}\right\rangle$, and (d) $\left\langle\tau_{1}\right\rangle,\left\langle\tau_{2 \mathrm{E}}\right\rangle,\left\langle\tau_{2 \mathrm{P}}\right\rangle$ as a function of $D$, plotted under the $N$-fixed condition. The chain length $N$ can be read in the legends where $N_{i}=2^{i}$.

$\phi_{0}=\phi_{0,1} \equiv 0.15$. Decreasing $\phi_{0}$ moves upward the curve in a parallel manner. The curve is then "reflected" toward right after meeting the demarcating line $D^{1.95(8)}$, and the scaling changes to $D^{3.06(2)}$. The results are consistent with the ones in Figure 14(c) because $\phi_{0} \sim N\left(\frac{\sigma}{D}\right)^{2}$ which yields the scaling exponent for $D$ about twice of the one for $N$ at a given $\phi_{0}$ value. Similarly, the ejection time $\left\langle\tau_{\text {ej }}\right\rangle$ scales like $D^{3.55(3)}$ at large $\phi_{0}$ and $D^{4.96(3)}$ at small $\phi_{0}$. The two exponents are about twice of the ones found in Figure 14(c). The leaving time $\left\langle\tau_{\text {leav }}\right\rangle$ stays very small and is about constant with varying $D$.

The components of the ejection time in Panel (b) show the following scaling behaviors: $\left\langle\tau_{1}\right\rangle \sim D^{3.58(3)}$ (existed when $\phi_{0}$ is large), $\left\langle\tau_{2 \mathrm{E}}\right\rangle \sim D^{5.19(8)}$ (existed when $\phi_{0}$ is small), and 
$\left\langle\tau_{2 \mathrm{P}}\right\rangle \sim D^{3.44(5)}$ at large $\phi_{0}$ and $D^{5.01(4)}$ at small $\phi_{0}$. The exponents are found to be the double of the ones in Figure $14(\mathrm{~d})$.

Figure 15(c) reveals that $\left\langle\tau_{\text {ent }}\right\rangle$ grows faster than a power law when $D$ is smaller than a critical value $D_{*} \sim \sigma N^{\nu}$ and becomes horizontal when $D>D_{*}$, if $N$ is fixed. The scaling line $D^{1.95(8)}$ in the figure tells how $\left\langle\tau_{\text {ent }}\right\rangle$ varies at the critical diameter. The ejection time $\left\langle\tau_{\mathrm{ej}}\right\rangle$ grows initial as $D^{2.68(5)}$, following the predicted behavior $D^{2 / \nu}$, and turns to becomes horizontal as $D>D_{*}$. Panel $(\mathrm{d})$ shows that the component $\left\langle\tau_{1}\right\rangle$ exhibits a $D^{2.68(5)}$ scaling. $\left\langle\tau_{2 E}\right\rangle$ is about constant in the concerned large $D$ region and $\left\langle\tau_{2 P}\right\rangle$ possesses the exponent value 2.98(5) for $D<D_{*}$ and turns to be leveled-off for $D>D_{*}$. The previous exponent is close to the theoretical value $\frac{1+z_{2 \mathrm{P}}}{\nu}$.

Figure 16 presents the variations of the different time components against the initial volume fraction $\phi_{0}$. With $N$ being fixed, Panels (a) and (b) are, in fact, the replots of Figure $15(\mathrm{c})$ and $15(\mathrm{~d})$ through the abscissa mapping $\phi_{0} \simeq N(\sigma / D)^{2}$. In the plots, $\left\langle\tau_{\text {ent }}\right\rangle$ is flat in the small $\phi_{0}$ region and decreases quickly as $\phi_{0}$ passes a critical value $\phi_{0 *}$. The curves join a master dropping curve and become zero (minus infinity in the log scale) at $\phi_{0} \simeq \phi_{\mathrm{p}}$. $\left\langle\tau_{\mathrm{ej}}\right\rangle$ shows a power-law decreasing behavior $\phi_{0}^{-1.32(5)}$ as $\phi_{0}>\phi_{0 *}$. In the confined stage, we observe the scaling $\left\langle\tau_{1}\right\rangle \sim \phi_{0}^{-1.35(4)}$. In the non-confined stage, $\left\langle\tau_{2 \mathrm{E}}\right\rangle$ is about constant while $\left\langle\tau_{2 \mathrm{P}}\right\rangle$ changes the scaling from $N^{1+z_{2 \mathrm{P}}}$ (independent of $\left.\phi_{0}\right)$ to be $N_{*}^{1+z_{2 \mathrm{P}}} \sim\left(N / \phi_{0}\right)^{\left(1+z_{2 \mathrm{P}}\right) /(d \nu)}$ when passing the critical point. The obtained exponent $-1.50(4)$ for $\phi_{0}$ is somewhat larger than the predicted value $-\frac{1+z_{2 \mathrm{P}}}{2 \nu} \simeq-1.67$.

The plots in Figure 16(c) and 16(d) can be related to Figure 14(a) and 14(b) via the same mapping $\phi_{0} \simeq N(\sigma / D)^{2}$ but it is the $D$ value being fixed. Therefore, the scaling exponents extracted from the two sets of figures should be identical. We can see that $\left\langle\tau_{\text {ent }}\right\rangle$ increases with $\phi_{0}$ with a consistent exponent 1.53(3) for $\phi_{0}<\phi_{0 *}$. In the region $\phi_{0}>\phi_{0 *}$, the decreasing curves collapse and tend to be zero at $\phi_{0}$ around 0.3 . $\left\langle\tau_{\mathrm{ej}}\right\rangle$ shows $\phi_{0}^{2.54(3)}$ for the cases experiencing only the non-confined stage, while it changes to $\phi_{0}^{0.52(5)}$ for the ones experiencing the confined and then non-confined stages. The scaling exponents for $\left\langle\tau_{1}\right\rangle$ and $\left\langle\tau_{2 \mathrm{E}}\right\rangle$ are $0.46(6)$ and $2.54(6)$, respectively. The $\left\langle\tau_{2 \mathrm{P}}\right\rangle$ data show consistent scaling $\phi_{0}^{2.49(5)}$ and tend to be leveled off as $\phi_{0}$ is large.

As seen in the above figures, the leaving time $\left\langle\tau_{\text {leav }}\right\rangle$ does not change basically with varying the $N, D$, and $\phi_{0}$ variables. It is because the relevant quantity, the pore length $\ell_{\mathrm{p}}$, is fixed in the study. To explore the behavior of $\left\langle\tau_{\text {leav }}\right\rangle$, we have performed simulations for $N=32$ 


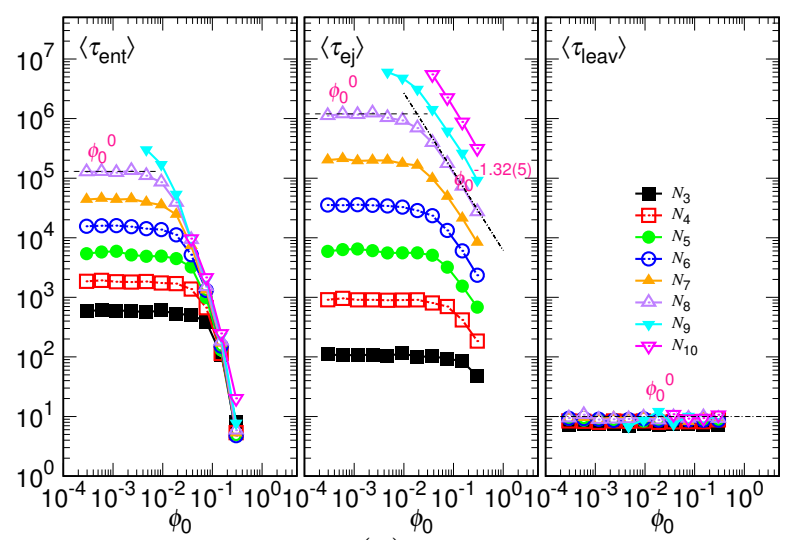

(a)

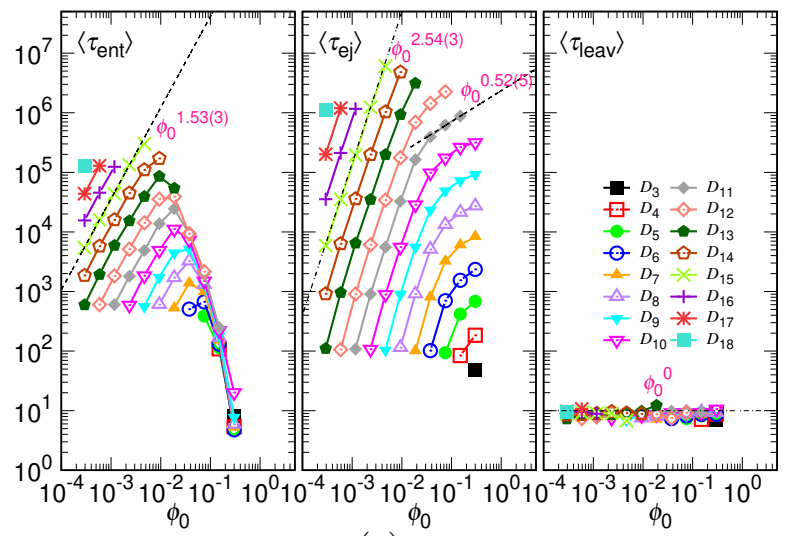

(c)

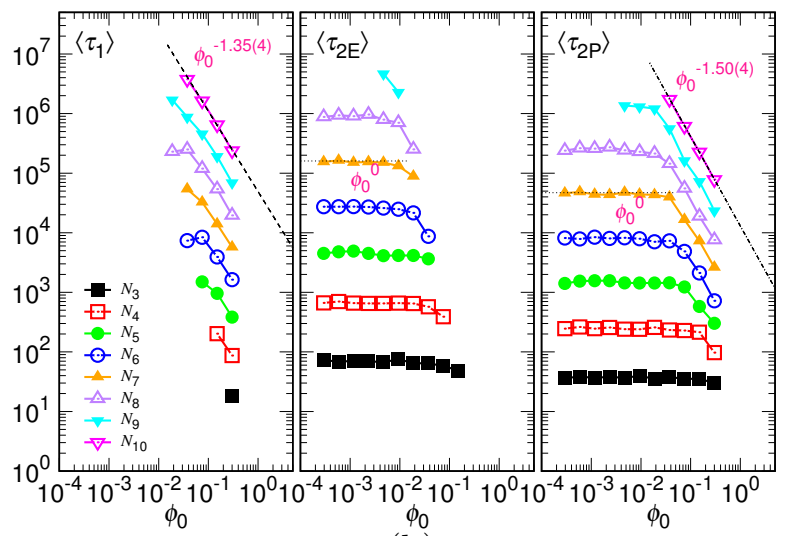

(b)

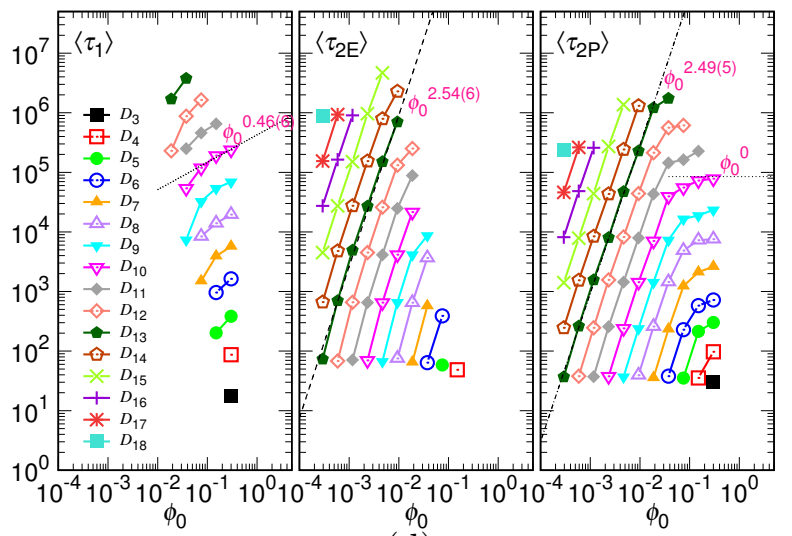

(d)

FIG. 16. (a) $\left\langle\tau_{\text {ent }}\right\rangle,\left\langle\tau_{\text {ej }}\right\rangle,\left\langle\tau_{\text {leav }}\right\rangle$, and (b) $\left\langle\tau_{1}\right\rangle,\left\langle\tau_{2 \mathrm{E}}\right\rangle,\left\langle\tau_{2 \mathrm{P}}\right\rangle$ as a function of $\phi_{0}$, plotted under the $N$-fixed condition. The chain length $N$ is indicated in the legends with $N_{i}=2^{i}$. (c) $\left\langle\tau_{\text {ent }}\right\rangle,\left\langle\tau_{\text {ej }}\right\rangle$, $\left\langle\tau_{\text {leav }}\right\rangle$, and $(\mathrm{d})\left\langle\tau_{1}\right\rangle,\left\langle\tau_{2 \mathrm{E}}\right\rangle,\left\langle\tau_{2 \mathrm{P}}\right\rangle$ as a function of $\phi_{0}$, plotted under the $D$-fixed condition. The $D$ value can be found in the legends, calculated by Eq. 7 .

by varying $\ell_{\mathrm{p}}$. The results are shown in Figure 17 .

We can see in Panel (a) that the leaving time increases with the pore length at various $\phi_{0}$ values and tends to grow as $\ell_{\mathrm{p}}^{1.14(8)}$. Panel (b) reveals that the $\left\langle\tau_{\text {leav }}\right\rangle$ value is basically independent of the initial volume fraction $\phi_{0}$. The reason for the properties is explained below. At the final moment of an ejection process (the leaving stage), the chain tail is driven across the pore channel by the chemical potential difference $\Delta \mu_{\mathrm{ps}}$ between the pore and the outer semi-space. The initial volume fraction $\phi_{0}$ thus has no effect on the leaving mechanism. The leaving time is predicted to be about $\Delta \mathfrak{t}_{0}\left(\frac{k_{\mathrm{B}} T}{\left|\Delta \mu_{\mathrm{ps}}\right| \sigma}\right) \ell_{\mathrm{p}}$, which is proportional to the pore length. What we have observed agrees mainly with the prediction. 


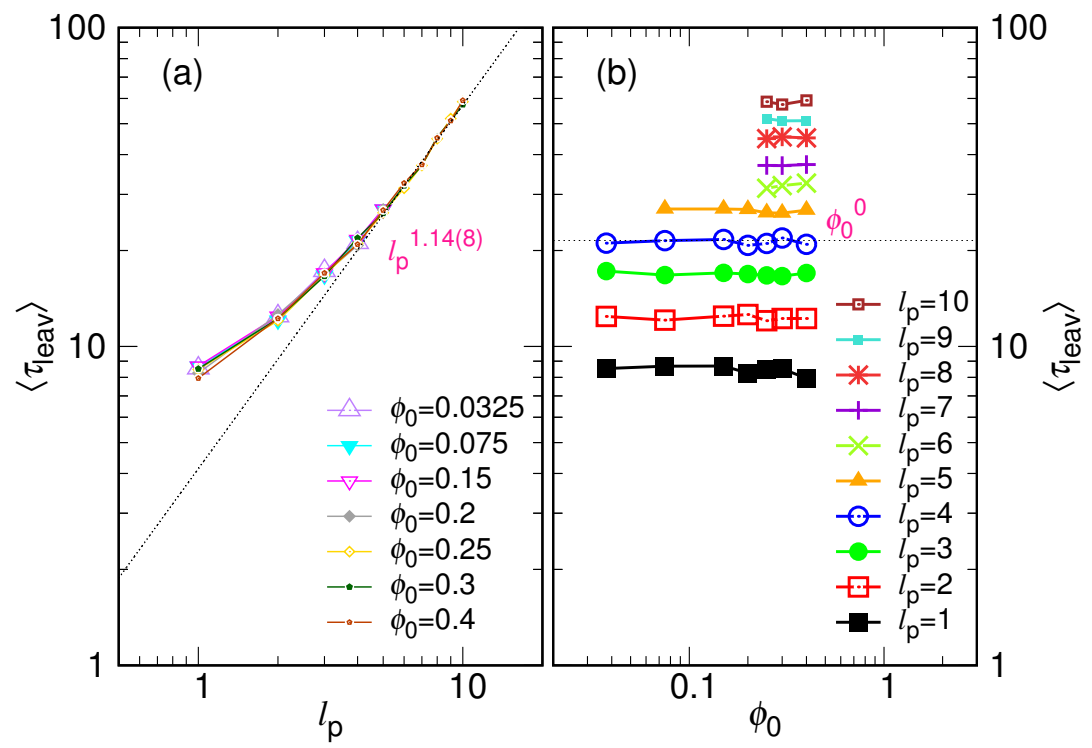

FIG. 17. (a) $\left\langle\tau_{\text {leav }}\right\rangle$ as a function of $\ell_{\mathrm{p}}$ at various $\phi_{0}$ values, indicated in the legend. (b) $\left\langle\tau_{\text {leav }}\right\rangle$ vs. $\phi_{0}$ with $\ell_{\mathrm{p}}$ being fixed. The chain length is 32 .

\section{DISCUSSIONS AND CONCLUSIONS}

To make connection with applications, we present time variational curves of the ejection coordinate $s$ in Figure 18. Because the processing time $\tau$ of an ejection varies a lot from one process to the others, the curves are plotted by using the normalized time and ejection coordinates, $t / \tau$ and $s / N$, for comparison. Five typical curves, together with the mean curve averaged over 500 independent runs, are presented at the three selected initial volume fractions.

At the large volume fraction, $\phi_{0}=\phi_{0,0}=0.3$, the differences between individual curves is relatively small (refer to Panel (a)). The ejection follows mainly the path of the mean curve. In other words, the evolution of the ejection is quite deterministic. As $\phi_{0}$ decreases, the evolution becomes more and more stochastic. The random-walk nature of the process leads to large fluctuations, upward and downward in the $s$-coordinate as shown in Panel (b). At zero initial volume fraction, $\phi_{0}=\phi_{0, \infty}$, a large free energy barrier has to be overcome by the system. The chain struggles for leaving the cis side. We can see that in addition to the large variation, the curve bounces so many times with the bottom line $s=0$, until the chain finds a way to go out of the trap and finally reaches the trans side.

In applications, a large initial volume fraction is generally preferable so that the ejection 


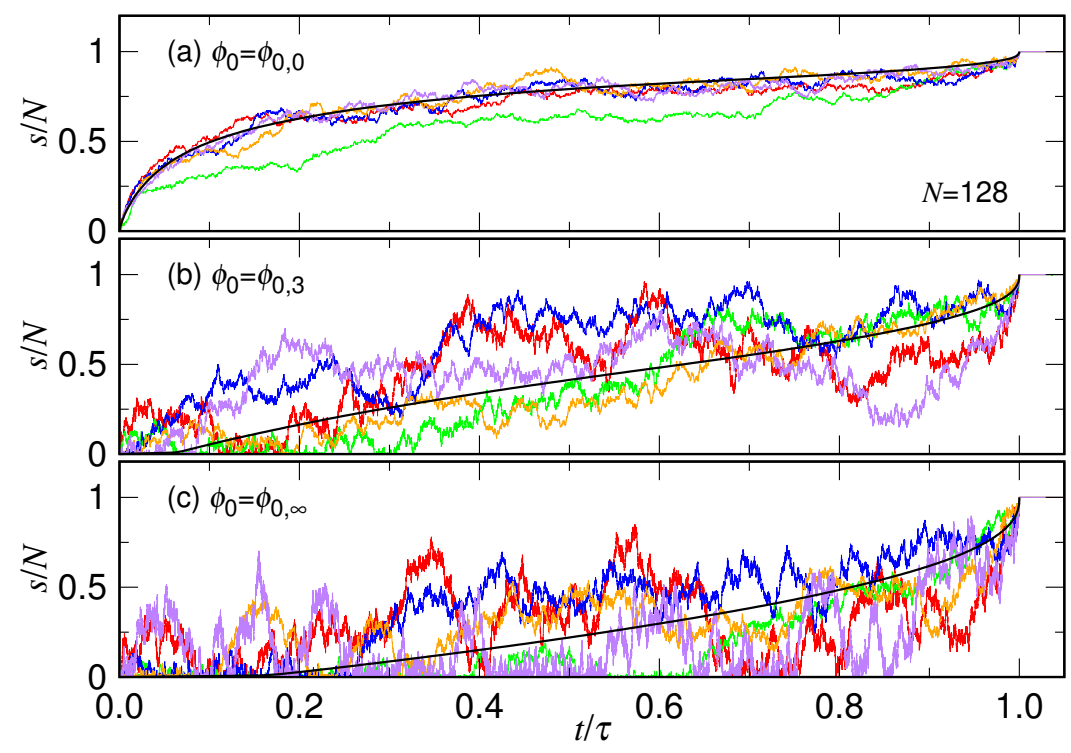

FIG. 18. Evolution of the normalized coordinate $s / N$ versus the normalize time $t / \tau$ in five typical ejection processes, plotted in colored curves, at (a) $\phi_{0}=\phi_{0,0}$, (b) $\phi_{0}=\phi_{0,3}$, and (c) $\phi_{0}=\phi_{0, \infty}$. The chain length is $N=128$. The mean evolution is plotted on top of the five curves in black color in each panel.

can be effectuated in a controllable (deterministic) way. Figure 18(a) has revealed that an individual ejection spends the first $20 \%$ of the time on ejecting about two thirds of the chain out of the cavity and the rest the remaining $80 \%$ of the time to complete it. The proportion of the chain to be ejected in the first $20 \%$ time is even higher if the chain length $N$ becomes longer by keeping the same $\phi_{0}$ value. A process like this definitely gives us a sensation that the ejection is drastically slowed down or seemly "halted" after spitting out a considerable long segment from the confining cavity. It might explain the experimental observations for the inhibition of DNA ejection from bacteriophages under certain conditions [12, 24, 56]. In particular, increasing the osmotic pressure from the outer solution could be regarded as an effective reduction of the osmotic pressure difference between the inside and outside of the cavity. Thus, the ejection might show an evolution curve similar to the small $\phi_{0}$ cases given in Panels (b) and (c) of the figure.

In this study, we have generalized the ejection theory for polymer to a two-dimensional space. The kinetics equation was derived from the Onsager's variational principle by using the free energy obtained in the scaling theory of polymer physics. The ejection speed was shown to scale as $m^{z_{1}} /\left(N^{x_{1}} N_{*}^{z_{1}+1}\right)$ in the confined stage and $m^{-z_{2 P}}$ in the non-confined stage 
with $x_{1}=1 / d, z_{1}=1 /(d \nu-1)$, and $z_{2 \mathrm{P}}=2 \nu$, where $m$ is the number of monomers in the circular cavity and $N_{*}$ is the critical number of monomers to distinguish the confined and the non-confined stages at a given cavity diameter $D$. The analysis showed that the non-confined stages can be further divided into two substages, determined by whether the chain length in the cavity is still longer than the one in the outer space or not. The spending time can be calculated in these stages and the ejection time $\tau_{\mathrm{ej}}$ is equal to $\tau_{1}+\tau_{2 \mathrm{E}}+\tau_{2 \mathrm{P}}$. Because the pore has finite length, non-negligible time is required for the head monomer to traverse the pore channel to be able to start ejecting chain segments into the outer space. The time spent for the tail monomer to go across the pore channel to end the process is generally short. The two events define two additional stages before and after the main ejection stage, called the entering stage and the leaving stage. In the entering stage, $\tau_{\text {ent }}$ can be calculated by solving the Kramers escape problem while $\tau_{\text {leav }}$ for the leaving stage is derived from the chemical potential difference. The total processing time $\tau$ is thus the sum of the three components, $\tau_{\text {ent }}, \tau_{\text {ej }}$, and $\tau_{\text {leav }}$. Each has its own scaling behavior. In the entering stage, $\tau_{\text {ent }}$ is described by Eq. S3 in SI. It exhibits an exponential increase with the pore length, like $\eta \exp \left(\frac{\ell_{\mathrm{p}} \Delta \mu_{\mathrm{cp}}}{\sigma k_{\mathrm{B}} T}\right)$, for $\phi_{0}$ much smaller than $\phi_{\mathrm{p}}$, the local volume fraction of a monomer presented in the pore channel. If $\phi_{0}$ is around $\phi_{\mathrm{p}}, \tau_{\text {ent }}$ is characterized by a diffusive time $\frac{\eta \ell_{\mathrm{p}}^{2}}{2 k_{\mathrm{B}} T}$. In the main ejection stage, if the confined stage is the dominated contribution, $\tau_{\mathrm{ej}}$ scales as $N^{x_{1}+(2 / d \nu)} \phi_{0}^{-2 / d \nu}$, or equivalently $N^{(2 d+7) / 3 d} \phi_{0}^{-(2 d+4) / 3 d}$ by replacing $\nu$ with $3 /(d+2)$. If the dominating is the non-confined stage, the scaling of $\tau_{\mathrm{ej}}$ is a combination of the two terms, $N^{2+x_{1}}$ and $N^{1+z_{2 \mathrm{P}}}$, which can be expressed as a function of $d$ as $N^{2+(1 / d)}$ and $N^{(d+8) /(d+2)}$, respectively. In the leaving stage, the spending time is $\tau_{\text {leav }} \sim \ell_{\mathrm{p}}\left(\frac{k_{\mathrm{B}} T}{\sigma\left|\Delta \mu_{\mathrm{ps}}\right|}\right)$.

Elaborated molecular dynamics simulations have been performed to verify the generalized ejection theory in the two-dimensional space. By varying the chain length $N$, the cavity diameter $D$, and thus the initial volume fraction $\phi_{0}$, we demonstrated that the ejection speed can be well described by the kinetics equation Eq.11 with the scaling exponents in consistent with the predictions (Figures 3 to 7 ). The study for the local minimum of the ejection speed did show that the critical number $N_{*}$ varies as $D^{1 / \nu}$ with $\nu=0.75$ being the two-dimensional Flory exponent (Figure 8). The time evolution of $m$ revealed that a nucleation-like stage occurs prior to the main ejection stage. The trimmed curves were described by Eq. 12 and Eq. 13, respectively, when $m$ approaches the starting and the ending point of the ejection stage, with the exponents $\zeta_{1}=1.0$ and $\zeta_{2}=0.4$ (Figures 9 to 11). The entering time was then 
examined by varying the pore length, and shown to fulfill the properties of the first passage time obtained from the Kramers escape problem (Figures 12 and 13). The total processing time was decomposed in Figures 14, 15, 16 and the scaling behaviors were verified carefully in each stage by varying $N, D, \phi_{0}$. The results support the predicted behaviors given in TableIf. Finally, the leaving time was checked and demonstrated linearly proportional to the

pore length (Figure 17). Owing to the consistency of the various scaling behaviors in the different stages, we conclude that the free energy landscape of ejection should be as sketched as in Figure 1.

The ejection theory has been demonstrated valid both in this two-dimensional study and in the previous three-dimensional investigation [39, 40]. It is a very robust theory able to predict variations of various quantities such as the ejection speed, the ejection coordinate, and the ejection time. A process starting with a high density as well as with zero volume fraction can be well explained under the same theoretical framework. In other words, the theory can be used to understand the phenomena of polymer ejection and the polymer translocation across a wall. It shows how complicated the kinetics can be in releasing a confined or non-confined polymer through a channel. It allows people to get insight of the ejection physics and helps researchers in the development of various ejection devices for two-dimensional or three-dimensional applications.

\section{ACKNOWLEDGMENTS}

This material is based upon work supported by the Ministry of Science and Technology, Taiwan under the contract No. MOST 109-2112-M-007-016.

\section{REFERENCES}

[1] T. Vo-Dinh, ed., Nanotechnology in Biology and Medicine (CRC Press, 2017).

[2] T. Nagamune, Biomolecular engineering for nanobio/bionanotechnology, Nano Converg. 4, 9 $(2017)$.

[3] Y.-C. Lee and J.-Y. Moon, Introduction to Bionanotechnology (Springer Singapore, 2020). 
[4] X. Liu, M. M. Skanata, and D. Stein, Entropic cages for trapping DNA near a nanopore, Nat. Commun. 6, 6222 (2015).

[5] P. Cadinu, G. Campolo, S. Pud, W. Yang, J. B. Edel, C. Dekker, and A. P. Ivanov, Double barrel nanopores as a new tool for controlling single-molecule transport, Nano Lett. 18, 2738 (2018).

[6] X. Liu, P. Zimny, Y. Zhang, A. Rana, R. Nagel, W. Reisner, and W. B. Dunbar, Flossing DNA in a dual nanopore device, Small 16, 1905379 (2019).

[7] A. Choudhary, H. Joshi, H.-Y. Chou, K. Sarthak, J. Wilson, C. Maffeo, and A. Aksimentiev, High-fidelity capture, threading, and infinite-depth sequencing of single DNA molecules with a double-nanopore system, ACS Nano 14, 15566 (2020).

[8] S. Bhaskar and S. Lim, Engineering protein nanocages as carriers for biomedical applications, NPG Asia Mater. 9, e371 (2017).

[9] A. G. Rottinghaus, M. B. Amrofell, and T. S. Moon, Biosensing in smart engineered probiotics, Biotechnol. J. 15, 1900319 (2020),

[10] G. P. C. Salmond and P. C. Fineran, A century of the phage: past, present and future, Nat. Rev. Microbiol. 13, 777 (2015).

[11] K. E. Kortright, B. K. Chan, J. L. Koff, and P. E. Turner, Phage therapy: A renewed approach to combat antibiotic-resistant bacteria, Cell Host Microbe 25, 219 (2019).

[12] P. K. Purohit, M. M. Inamdar, P. D. Grayson, T. M. Squires, J. Kondev, and R. Phillips, Forces during bacteriophage DNA packaging and ejection, Biophys. J. 88, 851 (2005).

[13] I. J. Molineux and D. Panja, Popping the cork: mechanisms of phage genome ejection, Nat. Rev. Microbiol. 11, 194 (2013).

[14] V. Zárybnický, Mechanism of t-even DNA ejection, J. Theoret. Biol. 22, 33 (1969).

[15] I. S. Gabashvili and A. Y. Grosberg, Dynamics of double stranded DNA reptation from bacteriophage, J. Biomol. Struct. Dyn. 9, 911 (1992).

[16] J. Kindt, S. Tzlil, A. Ben-Shaul, and W. M. Gelbart, DNA packaging and ejection forces in bacteriophage, Proc. Natl. Acad. Sci. U.S.A. 98, 13671 (2001).

[17] S. Tzlil, J. T. Kindt, W. M. Gelbart, and A. Ben-Shaul, Forces and pressures in DNA packaging and release from viral capsids, Biophys. J. 84, 1616 (2003).

[18] M. M. Inamdar, W. M. Gelbart, and R. Phillips, Dynamics of DNA ejection from bacteriophage, Biophys. J. 91, 411 (2006). 
[19] P. Grayson, A. Evilevitch, M. M. Inamdar, P. K. Purohit, W. M. Gelbart, C. M. Knobler, and R. Phillips, The effect of genome length on ejection forces in bacteriophage lambda, Virol. 348, 430 (2006).

[20] C. São-José, M. de Frutos, E. Raspaud, M. A. Santos, and P. Tavares, Pressure built by DNA packing inside virions: Enough to drive DNA ejection in vitro, largely insufficient for delivery into the bacterial cytoplasm, J. Mol. Biol. 374, 346 (2007).

[21] D. Löf, K. Schillén, B. Jönsson, and A. Evilevitch, Forces controlling the rate of DNA ejection from phage $\lambda$, J. Mol. Biol. 368, 55 (2007).

[22] R. J. Nap, A. L. Božič, I. Szleifer, and R. Podgornik, The role of solution conditions in the bacteriophage PP7 capsid charge regulation, Biophys. J. 107, 1970 (2014)

[23] A. Evilevitch, L. T. Fang, A. M. Yoffe, M. Castelnovo, D. C. Rau, V. A. Parsegian, W. M. Gelbart, and C. M. Knobler, Effects of salt concentrations and bending energy on the extent of ejection of phage genomes, Biophys. J. 94, 1110 (2008).

[24] A. Leforestier, S. Brasilès, M. de Frutos, E. Raspaud, L. Letellier, P. Tavares, and F. Livolant, Bacteriophage t5 DNA ejection under pressure, J. Mol. Biol. 384, 730 (2008).

[25] E. Jończyk, M. Kłak, R. Miedzybrodzki, and A. Górski, The influence of external factors on bacteriophages - review, Folia Microbiol. 56, 191 (2011).

[26] S. Marion and A. Šiber, Ejecting phage DNA against cellular turgor pressure, Biophys. J. 107, 1924 (2014).

[27] M. Muthukumar, Polymer escape through a nanopore, J. Chem. Phys. 118, 5174 (2003).

[28] C. Y. Kong and M. Muthukumar, Polymer translocation through a nanopore. II. excluded volume effect, J. Chem. Phys. 120, 3460 (2004).

[29] Y. Shen and L. Zhang, Free energy barrier for compact chains escaping from a small sphere, Polymer 48, 3593 (2007).

[30] S. Yang and A. V. Neimark, Adsorption-driven translocation of polymer chain into nanopores, J. Chem. Phys. 136, 214901 (2012).

[31] J. M. Polson and A. C. M. McCaffrey, Polymer translocation dynamics in the quasi-static limit, J. Chem. Phys. 138, 174902 (2013).

[32] L.-Z. Sun, M.-B. Luo, W.-P. Cao, and H. Li, Theoretical study on the polymer translocation into an attractive sphere, J. Chem. Phys. 149, 024901 (2018). 
[33] P.-G. de Gennes, Scaling Concepts in Polymer Physics (Cornell University Press (New York), 1979).

[34] M. Rubinstein and R. H. Colby, Polymer physics (Oxford University Press, New York, 2003).

[35] M. Muthukumar, Polymer translocation (CRC Press, 2011).

[36] M. Muthukumar, Translocation of a confined polymer through a hole, Phys. Rev. Lett. 86, 3188 (2001).

[37] A. Cacciuto and E. Luijten, Confinement-driven translocation of a flexible polymer, Phys. Rev. Lett. 96, 238104 (2006).

[38] T. Sakaue and N. Yoshinaga, Dynamics of polymer decompression: Expansion, unfolding, and ejection, Phys. Rev. Lett. 102, 148302 (2009).

[39] H.-C. Huang and P.-Y. Hsiao, Scaling behaviors of a polymer ejected from a cavity through a small pore, Physical Review Letters 123, 267801 (2019).

[40] P.-Y. Hsiao, Scaling theory of a polymer ejecting from a cavity into a semi-space, Polymers 12, $3014(2020)$.

[41] A. Milchev, Single-polymer dynamics under constraints: scaling theory and computer experiment, J. Phys. Condens. Matter 23, 103101 (2011).

[42] D. Panja, G. T. Barkema, and A. B. Kolomeisky, Through the eye of the needle: recent advances in understanding biopolymer translocation, J. Phys. Condens. Matter 25, 413101 (2013).

[43] V. V. Palyulin, T. Ala-Nissila, and R. Metzler, Polymer translocation: the first two decades and the recent diversification, Soft matter 10, 9016 (2014).

[44] H. Kramers, Brownian motion in a field of force and the diffusion model of chemical reactions, Physica 7, 284 (1940).

[45] P. Hänggi, P. Talkner, and M. Borkovec, Reaction-rate theory: fifty years after kramers, Rev. Mod. Phys. 62, 251 (1990).

[46] M. Doi, Onsager's variational principle in soft matter, Journal of Physics: Condensed Matter 23, 284118 (2011).

[47] M. Doi, Soft Matter Physics (Oxford University Press, 2013).

[48] T. Sakaue and E. Raphaël, Polymer chains in confined spaces and flow-injection problems: some remarks, Macromolecules 39, 2621 (2006).

[49] G. Jannink and J. des Cloizeaux, Polymers in solution, J. Phys. Condens. Matter 2, 1 (1990). 
[50] E. Eisenriegler, K. Kremer, and K. Binder, Adsorption of polymer chains at surfaces: Scaling and monte carlo analyses, J. Chem. Phys. 77, 6296 (1982).

[51] M. Muthukumar, Polymer translocation through a hole, J. Chem. Phys. 111, 10371 (1999).

[52] H. Meirovitch and S. Livne, Computer simulation of long polymers adsorbed on a surface. II. critical behavior of a single self-avoiding walk, J. Chem. Phys. 88, 4507 (1988).

[53] H. Meirovitch and I. Chang, Surface critical exponents of self-avoiding walks on a square lattice with an adsorbing linear boundary: A computer simulation study, Phys. Rev. E 48, 1960 (1993).

[54] S. Plimpton, Fast parallel algorithms for short-range molecular dynamics, J. Comput. Phys. 117, 1 (1995), refer also to LAMMPS website at http://lammps.sandia.gov/.

[55] J. Paturej, J.-U. Sommer, and T. Kreer, Universal equation of state for flexible polymers beyond the semidilute regime, Phys. Rev. Lett. 122, 087081 (2019).

[56] A. Evilevitch, L. Lavelle, C. M. Knobler, E. Raspaud, and W. M. Gelbart, Osmotic pressure inhibition of DNA ejection from phage, Proc. Natl. Acad. Sci. U.S.A. 100, 9292 (2003).

[57] C. W. Gardiner, Handbook of stochastic methods for physics, chemistry and the natural sciences, 3rd ed. (Springer-Verlag, Berlin, 2004).

\section{AUTHOR CONTRIBUTIONS}

P.-Y.H. deduced the theory, performed the simulations, analyzed the data, prepared the figures, and wrote the manuscript. W.-Y.C. ran the simulations and analyzed the data.

\section{COMPETING INTERESTS}

The authors declare no competing interests.

\section{ADDITIONAL INFORMATION}

Supplementary Information The online version contains supplementary material available at https://xxxx.Xxxx.Xxxx

Correspondence and requests for materials should be addressed to P.-Y.H. 


\section{Supplementary Files}

This is a list of supplementary files associated with this preprint. Click to download.

- Suppllnfov1.pdf 